\title{
An interacting system in population genetics
}

\author{
By
}

\author{
Tokuzo ShIgA
}

(Communicated by Prof. S. Watanabe, Dec. 13, 1978)

\section{Introduction}

The purpose of the present paper is to investigate an interacting system describing an evolution of gene frequencies in population genetics. In particular, we are interested in the effect of geographical structure in the process of evolution.

We shall first explain our interacting system. Let $S$ be a countable set. Each element $i$ of $S$ is called "a colony". We assume that there are two alleles $A$ and $B$ at each colony and denote by $x_{i}\left(1-x_{i}\right)$ the gene frequency of the $A$-allele (resp. the $B$-allele) for the colony $i \in S$. A time evolution of gene frequencies is caused by the following three factors;

(1) mutation in each colony,

(2) migration among colonies, and

(3) random sampling drift.

It is described by means of the following stochastic differential equation,

$$
d x_{i}(t)=a\left(x_{i}(t)\right) d B_{i}(t)+\left(v-(u+v) x_{i}(t)+\sum_{j \in S} q_{i j} x_{j}(t)\right) d t
$$

where

$$
\begin{aligned}
& a(y)=\frac{1}{\sqrt{2 N}} \sqrt{y(1-y)} \text { if } 0 \leqq y \leqq 1 \\
& =0 \quad \text { otherwise, }
\end{aligned}
$$

with a positive constant $N, u$ and $v$ are constants such that $u \geqq 0$ and $v \geqq 0, q_{i j}$ $(i, j \in S)$ are constants such that $q_{i j} \geqq 0$ for $i \neq j$ and $\sum_{j \in S} q_{i j}=0$ for every $i$, and $\left\{B_{i}(t)\right\}_{i \in S}$ is an independent system of one-dimensional Brownian motions. $N$ denotes the population size of each colony at random sampling, $u$ and $v$ denote mutation rates between $A$ and $B$, and for $i \neq j, q_{i j}$ denotes the migration rate from $j \in S$ to $i \in S$.

We assume

$$
\sup _{i \in S}\left|q_{i i}\right|<+\infty \text {. }
$$

Then it is known that if $0 \leqq x_{i}(0) \leqq 1$ for all $i \in S,(1.1)$ has a unique solution satisfying

$$
0 \leqq x_{i}(t) \leqq 1 \text { for all } i \in S \text { and } t \geqq 0 \text {, a.s. (cf. Shiga-Shimizu [10]) }
$$


Accordingly, (1.1) defines a diffusion process on $X=\left[\begin{array}{ll}0 & 1\end{array}\right]^{S}$, where $X$ is equipped with the product topology. This system is also described analytically as follows.

Let $C(X)$ be the set of all continuous functions on $X$ and let $C_{0}^{2}(X)$ be the set of all functions $f \in C(X)$ which depend only on finite number of coordinates of $X$ and are twice continuously differentiable. Then there exists a unique strongly continuous semi-group $\left\{T_{t}\right\}$ on $C(X)$ such that

$$
\begin{gathered}
T_{t} 1=1 \text { and } T_{t} f \geqq 0 \text { for every } f \in C(X) \text { satisfying } f \geqq 0, \text { and } \\
T_{t} f-f=\int_{0}^{t} T_{s} A f d s \text { for every } f \in C_{0}^{2}(X), \text { where } A f(\boldsymbol{x})= \\
\sum_{i \in S} \frac{1}{2} a^{2}\left(x_{i}\right) D_{i i}^{2} f(x)+\sum_{i \in S}\left(v-(u+v) x_{i}+\sum_{j \in S} q_{i j} x_{j}\right) D_{i} f(x) \\
\text { for } \quad \boldsymbol{x}=\left\{x_{i}\right\} \in X, D_{i}=\frac{\partial}{\partial x_{i}}, \text { and } D_{i i}^{2}=\frac{\partial^{2}}{\partial x_{1}^{2}} .
\end{gathered}
$$

In fact, $\left\{T_{t}\right\}$ is obtained by using the solution of (1.1). That is, denoting by $\{\boldsymbol{x}(t: \boldsymbol{x})\}$ the solution of (1.1) with the initial condition $x(0)=x$, we have $T_{t} f(x)=E[f(x(t: x))]$ for each $f \in C(X)$.

Such a system is called a continuous time stepping stone model. Discrete time models have been investigated by many authors. cf. Kimura-Weiss [5], Fleming-Su [1], Maruyama [8], Itazu [2], and so on. On the other hand Nagylaki [9] considered recently an continuous time one-dimensional model by means of continuous approximations in space. However there is no systematic study known to us concerning stationary states in either case.

In this paper we shall study stationary states and ergodic behaviors of this system.

Let $\mathscr{P}(X)$ be the set of all probability measures on $X$ endowed with the topology of weak convergence. Then $\mathscr{P}(X)$ is compact since $X$ is compact metrizable. Let us denote by $\left\{T_{t}^{*}\right\}$ the adjoint semi-group on $\mathscr{P}(X)$ induced by $\left\{T_{t}\right\}$ and denote by $\mathscr{S}$ the set of all fixed points of $\left\{T_{t}^{*}\right\}$, i.e. $\mathscr{S}=\left\{\mu \in \mathscr{P}(X) ; T_{t}^{*} \mu=\mu\right.$ for all $\left.t \geqq 0\right\}$. Each element of $\mathscr{S}$ is called a stationary state or an equiliblium state of $\left\{T_{t}\right\}$. Since $\mathscr{S}$ is a non-empty compact convex set, we shall investigate the structure of $\mathscr{S}_{\text {ex }}$, the set of all extremal elements of $\mathscr{S}$.

In $\S 2$ we shall obtain the following general result in the case with mutation.

Theorem 1.1 Suppose that $u+v>0$. Then there exists a unique $v \in \mathscr{P}(X)$ such that

(1.6) $\lim _{t \rightarrow \infty} T_{t}^{*} \delta_{x}=v$ holds for every $x \in X$, where $\delta_{x}$ stands for the point mass at $\boldsymbol{x} \in X$.

Thus, $\mathscr{S}$ is a singleton. In particular if $u=0$ and $v>0(u>0$ and $v=0)$, then $\nu=\delta_{1}$ (resp. $\delta_{\mathbf{0}}$ ) where $\mathbf{1} \in X$ and $\mathbf{0} \in X$ are defined by $\mathbf{1}=\left\{x_{i}=1\right\}$ and $\mathbf{0}=\left\{x_{i}=0\right\}$.

Next we shall investigate the case without mutation, that is, the case $u=v=0$. Let us introduce three cases classified by the condition on migration rates. For 
this, we prepare several definitions and notations. Let $Q=\left\{q_{i j}\right\}$ and set $P_{t}=e^{t} Q$. By (1.2) $P_{t}$ is well-defined and satisfies

$$
P_{t} \geqq 0, P_{t} 1=1 \text { and } \frac{d P_{t}}{d t}=P_{t} Q=Q P_{t} \text { for every } t \geqq 0 \text {. }
$$

Let us define a transition probability $P_{t} \otimes P_{t}$ on $S \times S$ by

(1.8) $P_{t} \otimes P_{t}(i, j)=P_{t}\left(i_{1}, j_{1}\right) P_{t}\left(i_{2}, j_{2}\right)$ for each $\vec{\imath}=\left(i_{1}, i_{2}\right) \in S \times S$ and $\vec{j}=\left(j_{1}, j_{2}\right)$ $\in S \times S$.

We denote by $\left(X_{t}, P_{i}^{(2)}\right)$ the continuous time Markov chain on $S \times S$ associated with $P_{t} \otimes P_{t}$. We assume that $Q=\left\{q_{i j}\right\}$ is irreducible and introduce the following classification.

Case I $\quad P_{i}^{(2)}\left[\int_{0}^{\infty} I_{\Delta_{2}}\left(X_{t}\right) d t=+\infty\right]=1 \quad$ for all $\quad i \in S \times S$,

Case II $\quad P_{i}^{(2)}\left[\int_{0}^{\infty} I_{\Delta_{2}}\left(X_{t}\right) d t<+\infty\right]=1 \quad$ for all $\quad \vec{i} \in S \times S$, and

Case III $0<P_{i}^{(2)}\left[\int_{0}^{\infty} I_{\Delta_{2}}\left(X_{t}\right) d t=+\infty\right]<1 \quad$ for all $i \in S \times S$,

where $\Delta_{2}=\left\{\left(i_{1}, i_{2}\right) \in S \times S \mid i_{1}=i_{2}\right\}$.

Since $Q$ is irreducible Case I, Case II, and Case III exhaust all possibilities.

In $\S 3$ we shall consider Case $I$ and obtain the following.

Theorem 1.2 Assume Case I. Then we have

(i) $\mathscr{S}_{\text {ex }}=\left\{\delta_{\mathbf{0}}, \delta_{\mathbf{1}}\right\}$,

and

(ii) for a $\mu \in \mathscr{P}(X), T_{t}^{*} \mu$ converges as $t \rightarrow \infty$ if and only if $\sum_{j \in S} P_{t}(i, j) \int \mu(d x) x_{j}$ converges as $t \rightarrow \infty$ for each $i \in S$.

If this condition is satisfied, $\lim _{t \rightarrow \infty} \sum_{j \in S} P_{t}(i, j) \int \mu(d x) x_{j}=\lambda$ is independent of $i$ and $\lim _{t \rightarrow \infty} T_{t}^{*} \mu=\lambda \delta_{1}+(1-\lambda) \delta_{0}$.

Results on Case $I I$ and Case III are closely related to $Q$-harmonic functions. A function $h$ defined on $S$ is said to be $Q$-harmonic if $\sum_{j \in S} q_{i j} h(j)=0$ for each $i \in S$. Let us denote by $\mathscr{H}$ the set of all $Q$-harmonic functions satisfying $0 \leqq h \leqq 1$. Each $h \in \mathscr{H}$ can be regarded as an element of $X$ and $\delta_{h}$ denotes the point mass at $h$. Then we have

Theorem 1.3 Assume Case II. Then,

(i) $\lim _{t \rightarrow \infty} T_{t}^{*} \delta_{h}=v_{h}$ exists for each $h \in \mathscr{H}$,

(ii) $\int v_{h}(d x) x_{i}=h(i)$ for each $h \in \mathscr{H}$ and $i \in S$, 
and

(iii) $\mathscr{S}_{\text {ex }}=\left\{v_{h} ; h \in \mathscr{H}\right\}$.

Theorem 1.4 Assume the same conditions as in Theorem 1.3. Let $\mu \in \mathscr{P}(X)$ and $h \in \mathscr{H}$. Then $\lim _{t \rightarrow \infty} T_{t}^{*} \mu=v_{h}$ if and only if

(1.10) $\sum_{j \in S} P_{t}(i, j) x_{j}{ }^{t \rightarrow \infty}$ converges to $h(i)$ in probability with respect to $\mu$ for each $i \in S$.

The above theorems will be obtained in $\S 4$ and $\S 5$. In $\S 6$ we shall study Case III and give an example which illustrates a different phenomenon from Case $I$ and Case II. In $\S 7$ we shall give some examples and remarks.

Finally it would be interesting to compare our results and methods with those of Liggett [6] in the theory of infinite particle system with interactions.

\section{Auxiliary Markov chains and the case with mutation}

Let $I$ be the set of all non-negative integer-valued functions $\alpha$ defined on $S$ satisfying $|\alpha|=\sum_{j \in S} \alpha_{i}<+\infty . \quad \alpha$ is denoted by $\varepsilon^{i}$ if $\alpha_{i}=1$ and $\alpha_{j}=0$ for $j \neq i$. For $\alpha \in I$ and $\beta \in I, \alpha+\beta \in I$ is defined by $(\alpha+\beta)_{i}=\alpha_{i}+\beta_{i}$ for each $i \in S$. If $\alpha_{i} \geqq \beta_{i}$ for all $i \in S$ we denote it by $\alpha \geqq \beta$, and in such case, $\alpha-\beta \in I$ is defined by $(\alpha-\beta)_{i}=\alpha_{i}-\beta_{i}$ for each $i \in S$.

Now, let us introduce two kinds of continuous time Markov chains on $I$. Set

$$
\begin{aligned}
& R_{\alpha, \beta}= \begin{cases}\alpha_{i} q_{i j} & \text { if } \beta=\alpha-\varepsilon^{i}+\varepsilon^{j} \in I \quad(i \neq j) \\
\frac{1}{4 N} \alpha_{i}\left(\alpha_{i}-1\right)+v \alpha_{i} & \text { if } \beta=\alpha-\varepsilon^{i} \in I \\
\sum_{i \in S} \alpha_{i} q_{i i}-\frac{1}{4 N} \sum_{i \in S} \alpha_{i}\left(\alpha_{i}-1\right)-v|\alpha| \text { if } \beta=\alpha \\
0 & \text { otherwise. }\end{cases} \\
& \tilde{R}_{\alpha, \beta}= \begin{cases}\alpha_{i} q_{i j} & \text { if } \beta=\alpha-\varepsilon^{i}+\varepsilon^{j} \in I \quad(i \neq j) \\
\sum_{i \in S} \alpha_{i} q_{i i} & \text { if } \beta=\alpha \\
0 & \text { otherwise. }\end{cases}
\end{aligned}
$$

Then it follows easily that under the condition (1.2) $R=\left\{R_{\alpha, \beta}\right\}$ (resp. $\widetilde{R}=\left\{\widetilde{R}_{\alpha, \beta}\right\}$ ) generates a unique continuous time conservative Markov chain $\left(\alpha_{t}, \boldsymbol{P}_{\alpha}\right)_{\alpha \in I}$ (resp. $\left.\left(\alpha_{t}, \tilde{\boldsymbol{P}}_{\alpha}\right)_{\alpha \in I}\right)$ on $I$ such that

$$
\begin{array}{ll}
\lim _{t \downarrow 0} \frac{\boldsymbol{P}_{\alpha}\left(\alpha_{t}=\beta\right)-I_{\alpha, \beta}}{t}=R_{\alpha, \beta} & \text { for } \quad \alpha, \beta \in I \\
\lim _{t \downarrow 0} \frac{\tilde{\boldsymbol{P}}_{\alpha}\left(\alpha_{t}=\beta\right)-I_{\alpha, \beta}}{t}=\widetilde{R}_{\alpha, \beta} & \text { for } \quad \alpha, \beta \in I
\end{array}
$$


where

$$
I_{\alpha, \beta}= \begin{cases}1 & \text { if } \alpha=\beta \\ 0 & \text { otherwise. }\end{cases}
$$

In fact, it is easy to see that $\left\{\alpha_{t}, \tilde{\boldsymbol{P}}_{\alpha}\right\}_{\alpha \in I}$ is an independent system of $|\alpha|$ number of $P_{t}$-Markov chains, i.e. it satisfies

$$
\tilde{\boldsymbol{E}}_{\alpha}\left[g\left(\alpha_{t}\right)\right]=\sum_{j_{1} \in S} \cdots \sum_{j_{m} \in S} \prod_{k=1}^{m} P_{t}\left(i_{k}, j_{k}\right) g\left(\varepsilon^{j_{1}}+\cdots+\varepsilon^{j_{m}}\right)
$$

if $\alpha=\varepsilon^{i_{1}}+\cdots+\varepsilon^{i_{m}}$, for every bounded function $g$ on $I$. In particular, if $v=0$ and $|\alpha|=1$, both $\left(\alpha_{t}, \boldsymbol{P}_{\alpha}\right)$ and $\left(\alpha_{t}, \tilde{\boldsymbol{P}}_{\alpha}\right)$ can be regarded as $P_{t}$-Markov chains. Let $\Delta$ $=\left\{\alpha \in I \mid \alpha_{i} \geqq 2\right.$ for some $\left.i \in S\right\}$. If $v=0,\left(\alpha_{t}, \boldsymbol{P}_{\alpha}\right)_{\alpha \in I}$ has the same probabilitic behavior as $\left(\alpha_{t}, \tilde{\boldsymbol{P}}_{\alpha}\right)_{\alpha \in I}$ up to the hitting time for $\Delta$. But when $\alpha$ lies on $\Delta$, there is a possibility that one of particles disappears.

Let $\eta_{n}$ be the $n$-th jumping time of $\left\{\alpha_{t}\right\}$, i.e. $\eta_{1}=\inf \left\{t ; \alpha_{t} \neq \alpha_{0}\right\}, \eta_{2}=\eta_{1}+\eta_{1}\left(\vartheta_{\eta_{1}}\right)$, $\ldots, \eta_{n}=\eta_{n-1}+\eta_{1}\left(\vartheta_{\eta_{n-1}}\right)$, where $\vartheta$ stands for the shift operator of the Markov chain $\left(\alpha_{t}, \boldsymbol{P}_{\alpha}\right)$ or $\left(\alpha_{t}, \tilde{\boldsymbol{P}}_{\alpha}\right)$. Then we have immediately the following.

Lemma 2.1 If $\alpha \in \Delta$, then

$$
\boldsymbol{P}_{\alpha}\left[\left|\alpha_{\eta_{1}}\right|=\left|\alpha_{0}\right|\right]=\frac{\sum_{i \in S} \alpha_{i}\left|q_{i i}\right|}{\sum_{i \in S} \alpha_{i}\left|q_{i i}\right|+\frac{1}{4 N} \sum_{i \in S} \alpha_{i}\left(\alpha_{i}-1\right)+v|\alpha|}
$$

For each $\alpha \in I$, we put $f_{\alpha}(\boldsymbol{x})=\prod_{i \in S} x_{i}^{\alpha_{i}}$ and $f_{0}(\boldsymbol{x}) \equiv 1$. Then we have the following.

Lemma 2.2 For $A$ defined in (1.5), it holds that

$$
A f_{\alpha}(\boldsymbol{x})=\sum_{\beta \in I} R_{\alpha, \beta} f_{\beta}(\boldsymbol{x})-u|\alpha| f_{\alpha}(\boldsymbol{x}) \quad \text { for } \quad \text { each } \alpha \in I .
$$

Proof. Noting that $D_{i} f_{\alpha}(\boldsymbol{x})=\alpha_{i} f_{\alpha-\varepsilon^{i}}(\boldsymbol{x})$ and $D_{i i}^{2} f_{\alpha}(\boldsymbol{x})=\alpha_{i}\left(\alpha_{i}-1\right) \times f_{\alpha-2 \varepsilon^{i}}(\boldsymbol{x})$, it follows easily that

$$
\begin{aligned}
A f_{\alpha}(\boldsymbol{x})= & \sum_{i \in S}\left(\frac{1}{4 N} \alpha_{i}\left(\alpha_{i}-1\right)+v \alpha_{i}\right)\left(f_{\alpha-\varepsilon^{i}}(\boldsymbol{x})-f_{\alpha}(\boldsymbol{x})\right)+ \\
& \sum_{i \in S} \sum_{j \in S} \alpha_{i} q_{i j} f_{\alpha-\varepsilon^{i}+\varepsilon^{j}}-u|\alpha| f_{\alpha}(\boldsymbol{x}) .
\end{aligned}
$$

The following lemma plays an essential role throughout this paper.

\section{Lemma 2.3}

$$
T_{t} f_{\alpha}(\boldsymbol{x})=\boldsymbol{E}_{\alpha}\left[f_{\alpha_{t}}(\boldsymbol{x}) \exp \left(-u \int_{0}^{t}\left|\alpha_{s}\right| d s\right)\right] \quad \text { for all } \alpha \in I .
$$

Proof. By (1.5) and Lemma 2.2, we see that for each $\alpha \in I$

$$
T_{t} f_{\alpha}(\boldsymbol{x})-f_{\alpha}(\boldsymbol{x})=\sum_{\beta \in I} R_{\alpha, \beta} \int_{0}^{t} T_{s} f_{\beta}(\boldsymbol{x}) d s-u|\alpha| T_{t} f_{\alpha}(\boldsymbol{x}) .
$$

Hence $g(t, \alpha)=T_{t} f_{\alpha}(x)$ satisfies the following equation, 


$$
\left\{\begin{array}{l}
\frac{d}{d t} g(t, \alpha)=\sum_{\beta \in I} R_{\alpha, \beta} g(t, \beta)-u|\alpha| g(t, \alpha) \\
g(0, \alpha)=f_{\alpha}(x)
\end{array}\right.
$$

On the other hand it is well-known that (2.8) is uniquely solved by means of the Feynman-Kac formula as follows: (cf. Ito [3])

$$
g(t, \alpha)=\boldsymbol{E}_{\alpha}\left[f_{\alpha_{t}}(\boldsymbol{x}) \exp \left(-u \int_{0}^{t}\left|\alpha_{s}\right| d s\right)\right]
$$

Thus we get (2.6).

Lemma 2.4 Let $\zeta_{0}=\inf \left\{t \geqq 0 ;\left|\alpha_{t}\right|=0\right\}$. Then

(i) if $v>0, \boldsymbol{P}_{\alpha}\left[\zeta_{0}<+\infty\right]=1$ for every $\alpha \in I$, and

(ii) if $v=0, \boldsymbol{P}_{\alpha}\left[\zeta_{0}=+\infty\right]=1$ for every $\alpha \neq 0 \in I$.

Proof. (ii) is trivial because of the definition of $\left(\alpha_{t}, \boldsymbol{P}_{\alpha}\right)$. By Lemma 2.1,

$$
\boldsymbol{P}_{\alpha}\left[\left|\alpha_{\eta_{1}}\right|=\left|\alpha_{0}\right|\right] \leqq \frac{\sup _{i}\left|q_{i i}\right|}{\sup _{i}\left|q_{i i}\right|+v} \equiv \lambda \text { for every } \alpha \in I .
$$

Let $\zeta=\inf \left\{t \geqq 0 ;\left|\alpha_{t}\right|<\left|\alpha_{0}\right|\right\}$. By the strong Markov property, we have $\boldsymbol{P}_{\alpha}[\zeta=$ $+\infty] \leqq \boldsymbol{P}_{\alpha}\left[\left|\alpha_{\eta_{1}}\right|=\left|\alpha_{\eta_{2}}\right|=\cdots=\left|\alpha_{\eta_{n}}\right|=\left|\alpha_{0}\right|\right] \leqq \lambda^{n} \rightarrow 0$ as $n \rightarrow \infty$. Hence $\boldsymbol{P}_{\alpha}[\zeta<+\infty]=1$ for every $\alpha \in I$ with $|\alpha| \geqq 1$, and (i) follows from this.

Now we can prove Theorem 1.1. Let $v>0$. Then it follows from Lemma 2.3 and Lemma 2.4 that

$$
\lim _{t \rightarrow \infty} T_{t} f_{\alpha}(\boldsymbol{x})=\boldsymbol{E}_{\alpha}\left[\exp \left(-u \int_{0}^{\zeta_{0}}\left|\alpha_{s}\right| d s\right)\right] \quad \text { for any } \quad \alpha \in I \text { and } \boldsymbol{x} \in X .
$$

Hence there exists a unique probability measure $v$ on $X$ such that $\lim _{t \rightarrow \infty} T_{t} f(x)=$ $\langle v, f\rangle$ for all $f \in C(X){ }^{1)}$

In particular if $u=0$, then $\lim _{t \rightarrow \infty} T_{t} f_{\alpha}(\boldsymbol{x})=1$ for every $\alpha \in I$, namely $v=\delta_{1}$.

On the other hand if $u>0$ and $v=0, \boldsymbol{P}_{\alpha}\left[\zeta_{0}=+\infty\right]=1$ by Lemma 2.4. Hence it follows that $\lim _{t \rightarrow \infty} T_{t} f_{\alpha}(\boldsymbol{x})=0$ for any $\alpha \in I$ with $\alpha \neq 0$ and $\boldsymbol{x} \in X$, namely $v=\delta_{\boldsymbol{o}}$. Therefore we complete the proof of Theorem 1.1.

Remark If $u>0$, we can easily see that

$$
\left|T_{t} f_{\alpha}(\boldsymbol{x})-\langle v, f\rangle\right| \leqq e^{-u t} \quad \text { for all } \alpha \in I \text { and } \boldsymbol{x} \in X \text {. }
$$

\section{Case I}

Hereafter we investigate the case without mutation, namely the case $u=v=0$.

1) $\langle\nu, f\rangle=\int \nu(d \boldsymbol{x}) f(\boldsymbol{x})$ 
Then $\left(\alpha_{t}, \boldsymbol{P}_{\alpha}\right)_{\alpha \in I}$ can be interpreted as follows: each particle in $\alpha$ moves independently according to the transition law $P_{t}$ until two particles occupy the same site in $S$. When more than two particles occupy the same site in $S$, one of them may disappear with positive probability at the next jumping time.

Let us introduce the following Markov times.

$$
\begin{aligned}
& \zeta=\inf \left\{t \geqq 0 ;\left|\alpha_{t}\right|<\left|\alpha_{0}\right|\right\}, \quad \zeta_{k}=\inf \left\{t \geqq 0 ;\left|\alpha_{t}\right| \leqq k\right\} \text { for } k=0,1, \ldots, \\
& \tau_{1}=\inf \left\{t \geqq 0 ; \alpha_{t} \in I \backslash \Delta, \text { and }\left|\alpha_{t}\right|=\left|\alpha_{0}\right|\right\}, \\
& \sigma_{1}=\inf \left\{t \geqq \tau_{1} ; \alpha_{t} \in \Delta\right\} \text { and } \tau_{n} \text { and } \sigma_{n} \text { are defined inductively by } \\
& \tau_{n}=\sigma_{n-1}+\tau_{1}\left(\vartheta_{\sigma_{n-1}}\right) \text { and } \sigma_{n}=\tau_{n}+\sigma_{1}\left(\vartheta_{\tau_{n}}\right), \text { where } \sigma_{0}=0 .
\end{aligned}
$$

\section{Lemma 3.1}

(i ) $T_{t} f_{\alpha}(\boldsymbol{x})=\boldsymbol{E}_{\alpha}\left[f_{\alpha_{t}}(\boldsymbol{x})\right]$ for every $\alpha \in I$ and $\boldsymbol{x} \in X$.

(ii) $\boldsymbol{E}_{\alpha}\left[g\left(\alpha_{t}\right) ; \sigma_{1}=+\infty\right]=\tilde{\boldsymbol{E}}_{\alpha}\left[g\left(\alpha_{t}\right) ; \sigma_{1}=+\infty\right]$ for every bounded function $g$ on I, if $\alpha \in I \backslash \Delta$.

(iii) For each integer $m \geqq 2$, there exists a constant $\lambda(m)<1$ such that $\boldsymbol{P}_{\alpha}\left[\left|\alpha_{\eta_{1}}\right|\right.$ $\left.=\left|\alpha_{0}\right|\right] \leqq \lambda(m)$ if $\alpha \in \Delta$ and $|\alpha|=m$.

(iv) $[\zeta=+\infty]=\left[\tau_{n}<+\infty, \sigma_{n}=+\infty\right.$ for some $\left.n \geqq 1\right]$ a.s. $\boldsymbol{P}_{\alpha}$.

Proof. (i), (ii) and (iii) follows from Lemma 2.1, Lemma 2.3 and the definition of the Markov chains. So we show only (iv). Let $\alpha \in \Delta$. Then

$$
\begin{aligned}
& \boldsymbol{P}_{\alpha}\left[\zeta=+\infty, \tau_{1}=+\infty\right] \leqq \boldsymbol{P}_{\alpha}\left[\alpha_{\eta_{1}} \in \Delta, \ldots, \alpha_{\eta_{n}} \in \Delta,\left|\alpha_{\eta_{n}}\right|=\left|\alpha_{0}\right|\right] \\
& \quad \leqq \boldsymbol{E}_{\alpha}\left[\boldsymbol{P}_{\alpha_{\eta_{n-1}}}\left[\left|\alpha_{\eta_{1}}\right|=\left|\alpha_{0}\right|\right] ; \alpha_{\eta_{1}} \in \Delta, \ldots, \alpha_{\eta_{n-1}} \in \Delta,\left|\alpha_{\eta_{n-1}}\right|=\left|\alpha_{0}\right|\right] \\
& \quad \leqq \lambda(|\alpha|) \boldsymbol{P}_{\alpha}\left[\alpha_{\eta_{1}} \in \Delta, \ldots, \alpha_{\eta_{n-1}} \in \Delta,\left|\alpha_{\eta_{n-1}}\right|=\left|\alpha_{0}\right|\right] \leqq \lambda(|\alpha|)^{n} .
\end{aligned}
$$

Hence we have

$$
\boldsymbol{P}_{\alpha}\left[\zeta=+\infty, \tau_{1}=+\infty\right]=0
$$

Also,

$$
\begin{aligned}
& \boldsymbol{P}_{\alpha}\left[\zeta=+\infty \mid \tau_{n}<+\infty, \sigma_{n}=+\infty \text { for some } n \geqq 1\right] \\
& \quad=\boldsymbol{P}_{\alpha}\left[\zeta=+\infty, \sigma_{n-1}<+\infty, \tau_{n}=+\infty \text { for some } n \geqq 1\right]+\boldsymbol{P}_{\alpha}\left[\sigma_{n}<+\infty \text { for all } n\right]
\end{aligned}
$$

the first term $=\sum_{n=1}^{\infty} \boldsymbol{E}_{\alpha}\left[\boldsymbol{P}_{\alpha_{\sigma_{n-1}}}\left[\zeta=+\infty, \tau_{1}=+\infty\right] ; \sigma_{n-1}<+\infty\right]=0$

by (3.1), and

the second term $=\boldsymbol{P}_{\alpha}\left[\sigma_{n}<+\infty\right]=\boldsymbol{E}_{\alpha}\left[\boldsymbol{P}_{\alpha_{\sigma_{n-1}}}\left[\sigma_{1}<+\infty\right] ; \sigma_{n-1}<+\infty\right]$

$$
\begin{aligned}
& \leqq \boldsymbol{E}_{\alpha}\left[\boldsymbol{P}_{\alpha_{\sigma_{n-1}}}\left[\left|\alpha_{\eta_{1}}\right|=\left|\alpha_{0}\right|\right] ; \sigma_{n-1}<+\infty\right] \\
& \leqq \lambda(|\alpha|) \boldsymbol{P}_{\alpha}\left[\sigma_{n-1}<+\infty\right] \leqq \lambda(|\alpha|)^{n} \longrightarrow 0 \text { as } n \longrightarrow \infty
\end{aligned}
$$

Thus we obtain $[\zeta=+\infty] \subset\left[\tau_{n}<+\infty, \sigma_{n}=+\infty\right.$ for some $\left.n \geqq 1\right] \boldsymbol{P}_{\alpha}$-a.s. . But the converse inclusion is trivial. 
Lemma 3.2 The following (i), (ii) and (iii) are mutually equivalent.

(i) $Q=\left\{q_{i j}\right\}$ satisfies the condition of Case $I$.

(ii) $\tilde{\boldsymbol{P}}_{\alpha}\left[\sigma_{n}<+\infty\right.$ for every $\left.n \geqq 1\right]=1$ for every $\alpha \in I$ with $|\alpha|=2$.

(iii) $\boldsymbol{P}_{\alpha}\left[\zeta_{1}<+\infty\right]=1$ for every $\alpha \in I$.

Proof. "(i) $\rightarrow($ ii)" is trivial. Next let us assume (ii) and let $|\alpha|=2$. Then

$\tilde{\boldsymbol{E}}_{\alpha}\left[\exp \left(-\sum_{n=1}^{\infty}\left(\tau_{n+1}-\sigma_{n}\right)\right) ; \sigma_{n}<+\infty\right.$ for every $\left.n \geqq 1\right]$

$=\tilde{\boldsymbol{E}}_{\alpha}\left[\exp \left(-\left(\tau_{2}-\sigma_{1}\right)\right) \tilde{\boldsymbol{E}}_{\alpha_{\tau_{2}}}\left[\exp \left(-\sum_{n=1}^{\infty}\left(\tau_{n+1}-\sigma_{n}\right)\right) ; \sigma_{n}<+\infty\right.\right.$ for all $\left.n\right] ;$

$\left.\tau_{2}<+\infty\right]$

$\leqq \sup _{|\alpha|=2} \tilde{\boldsymbol{E}}_{\alpha}\left[\exp \left(\sum_{n=1}^{\infty}\left(\tau_{n+1}-\sigma_{n}\right)\right) ; \sigma_{n}<+\infty\right.$ for all $\left.n\right] \times$

$\sup _{|\alpha|=2} \tilde{\boldsymbol{E}}_{\alpha}\left[\exp \left(-\left(\tau_{2}-\sigma_{1}\right)\right)\right]$

Also for $|\alpha|=2$

$$
\begin{aligned}
& \tilde{\boldsymbol{E}}_{\alpha}\left[\exp \left(-\left(\tau_{2}-\sigma_{1}\right)\right)\right]=\tilde{\boldsymbol{E}}_{\alpha}\left[\tilde{\boldsymbol{E}}_{\alpha_{\sigma_{1}}}\left[e^{-\eta_{1}}\right] ; \sigma_{1}<+\infty\right] \leqq \sup _{|\alpha|=2} \frac{\left|\tilde{R}_{\alpha, \alpha}\right|}{1+\left|\tilde{R}_{\alpha, \alpha}\right|} \\
& \quad \leqq \frac{2 \sup _{i}\left|q_{i i}\right|}{1+2 \sup _{i}\left|q_{i i}\right|}<1
\end{aligned}
$$

Here we used the fact that the probability law of $\left(\eta_{1}, \tilde{\boldsymbol{P}}_{\alpha}\right)$ is the exponential distribution with parameter $\left|\widetilde{R}_{\alpha, \alpha}\right|$.

Hence we obtain

$$
\tilde{\boldsymbol{E}}_{\alpha}\left[\exp \left(-\sum_{n=1}^{\infty}\left(\tau_{n+1}-\sigma_{n}\right)\right)\right]=\tilde{\boldsymbol{E}}_{\alpha}\left[\exp \left(-\sum_{n=1}^{\infty}\left(\tau_{n+1}-\sigma_{n}\right)\right) ; \sigma_{n}<+\infty \text { for all } n\right]=0
$$

Accordingly we have

$$
\int_{0}^{\infty} I_{\Delta}\left(\alpha_{t}\right) d t=\sum_{n=1}^{\infty}\left(\tau_{n+1}-\sigma_{n}\right)=\infty \text { a.s. }\left(\tilde{\boldsymbol{P}}_{\alpha}\right) .
$$

Thus "(ii) $\rightarrow$ (i) is proved.

Next we shall show "(ii) $\rightarrow$ (iii)". First we note $\boldsymbol{P}_{\alpha}\left[\sigma_{1}=+\infty\right]=\tilde{\boldsymbol{P}}_{\alpha}\left[\sigma_{1}=+\infty\right]=0$, if $\alpha \in I \backslash \Delta$ and $|\alpha| \geqq 2$. By Lemma 3.1

$$
\begin{aligned}
\boldsymbol{P}_{\alpha}[\zeta & =+\infty]=\boldsymbol{P}_{\alpha}\left[\tau_{n}<+\infty, \sigma_{n}=+\infty \text { for some } n \geqq 1\right] \\
& =\sum_{n=1}^{\infty} \boldsymbol{E}_{\alpha}\left[\boldsymbol{P}_{\alpha_{\tau_{n}}}\left[\sigma_{1}=+\infty\right] ; \tau_{n}<+\infty\right]=0 .
\end{aligned}
$$

Hence $\boldsymbol{P}_{\alpha}[\zeta<+\infty]=1$ if $|\alpha| \geqq 2$. This implies (iii).

Conversely we assume that (iii) holds. For $\alpha \in I \backslash \Delta$ with $|\alpha|=2$, $\tilde{\boldsymbol{P}}_{\alpha}\left[\sigma_{1}<+\infty\right]$ $=\boldsymbol{P}_{\alpha}\left[\sigma_{1}<+\infty\right] \geqq \boldsymbol{P}_{\alpha}[\zeta<+\infty]=1$. 
(ii) follows from this.

Lemma 3.3 Assume Case I. Then every bounded Q-harmonic function is constant.

Proof. Let $h$ be any bounded $Q$-harmonic function. Then $P_{t} h=h$ for all $t \geqq 0$. Let us consider a continuous time Markov chain $\left(\left(X_{t}^{1}, X_{t}^{2}\right), P_{\left(i_{1}, i_{2}\right)}\right)$ on $S \times S$ such that

$$
\begin{cases}\text { (i) } \quad & E_{\left(i_{1}, i_{2}\right)}\left[g\left(X_{t}^{1}\right)\right]=P_{t} g\left(i_{1}\right) \text {, and } \\ & E_{\left(i_{1}, i_{2}\right)}\left[g\left(X_{t}^{2}\right)\right]=P_{t} g\left(i_{2}\right) \text { for any bounded function } g \text { on } S, \\ \text { (ii) } \quad & P_{\left(i_{1}, i_{2}\right)}[\xi<+\infty]=1 \text { for all }\left(i_{1}, i_{2}\right) \in S \times S \text {, and } \\ & P_{\left(i_{1}, i_{2}\right)}\left[X_{t}^{1}=X_{t}^{2} \text { for all } t \geqq \xi\right]=1, \\ & \text { where } \xi=\inf \left\{t \geqq 0 ; X_{t}^{1}=X_{t}^{2}\right\} .\end{cases}
$$

In fact, noting the condition of Case $I$, we can construct it as follows. Let $\left(\left(\bar{X}_{t}^{1}\right.\right.$, $\left.\left.\bar{X}_{t}^{2}\right), P_{\left(i_{1}, i_{2}\right)}\right)$ be a continuous time Markov chain on $S \times S$ with $P_{t} \otimes P_{t}$ as its transition probability. Then, $\bar{\xi}=\inf \left\{t \geqq 0 ; \bar{X}_{t}^{1}=\bar{X}_{t}^{2}\right\}<+\infty P_{\left(i_{1}, i_{2}\right)}-a . s$. Set $X_{t}^{1}=\bar{X}_{t}^{1}$, $X_{t}^{2}=\bar{X}_{t}^{2}$ for $t \leqq \xi$ and $X_{t}^{2}=\bar{X}_{t}^{1}$ for $t \geqq \xi$. Then $\left(\left(X_{t}^{1}, X_{t}^{2}\right), P_{\left(i_{1}, i_{2}\right)}\right)$ satisfies (3.2). Moreover, for $i_{1} \neq i_{2} \in S$,

$$
\begin{aligned}
\left|h\left(i_{1}\right)-h\left(i_{2}\right)\right| & =\left|E_{\left(i_{1}, i_{2}\right)}\left[h\left(X_{t}^{1}\right)-h\left(X_{t}^{2}\right)\right]\right| \\
& \leqq P_{\left(i_{1}, i_{2}\right)}[\xi>t] \cdot\|h\|_{\infty} \rightarrow 0 \text { as } t \rightarrow \infty .
\end{aligned}
$$

Thus we see that $h$ is constant.

\section{Proof of Theorem 1.2}

We shall prove only (ii) since (i) follows from (ii). Suppose that $\lim _{t \rightarrow \infty} T_{i}^{*} \mu$ exists for $\mu \in \mathscr{P}(X)$. Set

$$
m_{t}(\alpha)=\left\langle T_{t}^{*} \mu, f_{\alpha}\right\rangle=\left\langle\mu, T_{t} f_{\alpha}\right\rangle=\boldsymbol{E}_{\alpha}\left[\left\langle\mu, f_{\alpha_{t}}\right\rangle\right]=\boldsymbol{E}_{\alpha}\left[m_{0}\left(\alpha_{t}\right)\right] \text { (by Lemma 3.1). }
$$

Then $\lim _{t \rightarrow \infty} m_{t}(\alpha)=\bar{m}(\alpha)$ exists.

In particular, we see $\bar{m}\left(\varepsilon^{i}\right)=\lim _{t \rightarrow \infty} \sum_{j \in S} P_{t}(i, j) m_{0}\left(\varepsilon^{j}\right)$.

Since $\bar{m}\left(\varepsilon^{i}\right)$ is a $Q$-harmonic function as a function on $S, m\left(\varepsilon^{i}\right)=\lambda$ is independent of $i \in S$ by Lemma 3.3.

Thus we have

$$
\lim _{t \rightarrow \infty} m_{t}(\alpha)=\lambda \quad \text { if } \quad|\alpha|=1
$$

For $|\alpha| \geqq 1$,

$$
\begin{aligned}
m_{t}(\alpha) & =\boldsymbol{E}_{\alpha}\left[m_{0}\left(\alpha_{t}\right)\right]=\boldsymbol{E}_{\alpha}\left[m_{0}\left(\alpha_{t}\right) ; \zeta_{1}<t\right]+\boldsymbol{E}_{\alpha}\left[m_{0}\left(\alpha_{t}\right) ; \zeta_{1} \geqq t\right] \\
& =\boldsymbol{E}_{\alpha}\left[\left.\boldsymbol{E}_{\alpha_{\zeta_{1}}}\left[m_{0}\left(\alpha_{t-s}\right)\right]\right|_{s=\zeta_{1}} ; \zeta_{1}<t\right]+\boldsymbol{E}_{\alpha}\left[m_{0}\left(\alpha_{t}\right) ; \zeta_{1} \geqq t\right] .
\end{aligned}
$$


By using Lemma 3.2 and (3.3), we get

$$
\lim _{t \rightarrow \infty} m_{t}(\alpha)=\lambda \text { for all } \alpha \neq 0 .
$$

It follows immediately that

$$
\lim _{t \rightarrow \infty} T_{t}^{*} \mu=\lambda \delta_{1}+(1-\lambda) \delta_{\mathbf{o}} .
$$

We can also prove the converse by an analogous argument.

\section{Case II, Structure of $\mathscr{S}$}

In $\$ 4$ and $\S 5$ we always assume Case $I I$.

\section{Lemma 4.1}

(i) $\boldsymbol{P}_{\alpha}[\zeta<+\infty]<1$ for every $\alpha \in I$.

(ii) $\tilde{\boldsymbol{P}}_{\alpha}\left[\tau_{n}<+\infty\right.$, and $\sigma_{n}=+\infty$ for some $\left.n \geqq 1\right]=1$ for every $\alpha \in I$.

Proof. It follows easily from the irreducibility of $Q=\left\{q_{i j}\right\}$ that if $|\beta|=|\alpha|$, then $\boldsymbol{P}_{\alpha}\left[\alpha_{t}=\beta\right]>0$ for all $t>0$. So we have $\boldsymbol{P}_{\alpha}\left[\tau_{1}<+\infty\right]>0$ for each $\alpha \in I$. By the condition of Case II

$$
\tilde{\boldsymbol{P}}_{\alpha}\left[\int_{0}^{\infty} I_{\Delta}\left(\alpha_{t}\right) d t<+\infty\right]=1 \quad \text { for every } \quad \alpha \in I \text { with }|\alpha| \geqq 2 .
$$

This implies that $\tilde{\boldsymbol{P}}_{\alpha}\left[\sigma_{1}<+\infty\right]<1$ holds for every $\alpha \in I \backslash \Delta$ with $|\alpha| \geqq 2$, by an argument similar to Lemma 2.1 in Liggett's paper [6].

Hence we have

$$
\begin{aligned}
& \boldsymbol{P}_{\alpha}[\zeta=+\infty]=\boldsymbol{P}_{\alpha}\left[\tau_{1}<+\infty, \zeta=+\infty\right] \quad \text { (by Lemma 3.1) } \\
& \quad=\boldsymbol{E}_{\alpha}\left[\boldsymbol{P}_{\alpha_{\tau_{1}}}[\zeta=+\infty] ; \tau_{1}<+\infty\right] \geqq \boldsymbol{E}_{\alpha}\left[\tilde{\boldsymbol{P}}_{\alpha_{\tau_{1}}}\left[\sigma_{1}=+\infty\right] ; \tau_{1}<+\infty\right]>0 .
\end{aligned}
$$

(ii): In the proof of Lemma 3.2 we showed $\tilde{\boldsymbol{E}}_{\alpha}\left[\exp \left(-\int_{0}^{\infty} I_{\Delta}\left(\alpha_{t}\right) d t\right) ; \sigma_{n}<+\infty\right.$ for every $n \geqq 1]=0$ for every $\alpha \in I$ with $|\alpha|=2$. Since $\int_{0}^{\infty} I_{\Delta}\left(\alpha_{t}\right) \mathrm{d} t<\infty$ a.s. $\left(\tilde{\boldsymbol{P}}_{\alpha}\right)$ by the condition of Case $I I$, we see $\tilde{\boldsymbol{P}}_{\alpha}\left[\sigma_{n}<+\infty\right.$ for every $\left.n \geqq 1\right]=0$ for all $\alpha \in I$ with $|\alpha|=2$, and moreover this holds for all $\alpha \in I$ with $|\alpha| \geqq 2$. Thus we see that (ii) holds.

Now let us introduce the following spaces of harmonic functions.

$$
\begin{aligned}
& \mathscr{G}=\left\{g(\alpha) ; \text { defined on } I, 0 \leqq g \leqq 1 \text { and } \boldsymbol{E}_{\alpha}\left[g\left(\alpha_{t}\right)\right]=g(\alpha) \text { for all } \alpha \in I \text { and } t \geqq 0\right\} \\
& \widetilde{\mathscr{H}}=\left\{\tilde{h}(\alpha) ; \text { defined on } I, 0 \leqq \tilde{h} \leqq 1 \text { and } \tilde{\boldsymbol{E}}_{\alpha}\left[\tilde{h}\left(\alpha_{t}\right)\right]=\tilde{h}(\alpha) \text { for all } \alpha \in I \text { and } t \geqq 0\right\}
\end{aligned}
$$

In the following lemmas we shall establish a one-to-one correspondence between $\mathscr{G}$ and $\widetilde{\mathscr{H}}$.

Lemma 4.2 For every $\tilde{h} \in \tilde{\mathscr{H}}, \lim _{t \rightarrow \infty} \boldsymbol{E}_{\alpha}\left[\tilde{h}\left(\alpha_{t}\right)\right]$ exists. 
Proof. For $\alpha \in I \backslash \Delta$,

$$
\begin{aligned}
\tilde{\boldsymbol{E}}_{\alpha}\left[\tilde{h}\left(\alpha_{t}\right) ; \sigma_{1}=+\infty\right] & =\tilde{\boldsymbol{E}}_{\alpha}\left[\tilde{h}\left(\alpha_{t}\right) ; \sigma_{1}=+\infty\right] \\
& =\tilde{\boldsymbol{E}}_{\alpha}\left[\tilde{h}\left(\alpha_{t}\right)\right]-\tilde{\boldsymbol{E}}_{\alpha}\left[\tilde{h}\left(\alpha_{t}\right) ; \sigma_{1}<+\infty\right] \\
& =\tilde{h}(\alpha)-\tilde{\boldsymbol{E}}_{\alpha}\left[\tilde{h}\left(\alpha_{t}\right) ; \sigma_{1}<t\right]-\tilde{\boldsymbol{E}}_{\alpha}\left[\tilde{h}\left(\alpha_{t}\right) ; t \leqq \sigma_{1}<+\infty\right] .
\end{aligned}
$$

Since

$$
\tilde{\boldsymbol{E}}_{\alpha}\left[\tilde{h}\left(\alpha_{t}\right) ; \sigma_{1}<t\right]=\tilde{\boldsymbol{E}}_{\alpha}\left[\left.\tilde{\boldsymbol{E}}_{\alpha_{\sigma_{1}}}\left[\tilde{h}\left(\sigma_{t-s}\right)\right]\right|_{s=\sigma_{1}} ; \sigma_{1}<t\right]=\tilde{\boldsymbol{E}}_{\alpha}\left[\tilde{h}\left(\alpha_{\sigma_{1}}\right) ; \sigma_{1}<t\right],
$$

we have

$$
\lim _{t \rightarrow \infty} \boldsymbol{E}_{\alpha}\left[\tilde{h}\left(\alpha_{t}\right) ; \sigma_{1}=+\infty\right]=\tilde{h}(\alpha)-\tilde{\boldsymbol{E}}_{\alpha}\left[\tilde{h}\left(\alpha_{\sigma_{1}}\right) ; \sigma_{1}<+\infty\right]
$$

We denote the right-hand side of $(4.1)$ by $\tilde{h}_{1}(\alpha)$.

Then

$$
\begin{gathered}
\boldsymbol{E}_{\alpha}\left[\tilde{h}\left(\alpha_{t}\right) ; \zeta=+\infty\right]=\sum_{n=1}^{\infty} \boldsymbol{E}_{\alpha}\left[\tilde{h}\left(\alpha_{t}\right) ; \tau_{n}<+\infty, \sigma_{n}=+\infty\right] \\
=\sum_{n=1}^{\infty} \boldsymbol{E}_{\alpha}\left[\left.\boldsymbol{E}_{\alpha_{\tau_{n}}}\left[\tilde{h}\left(\alpha_{t-s}\right) ; \sigma_{1}=+\infty\right]\right|_{s=\tau_{n}} ; \tau_{n}<t\right]+ \\
\sum_{n=1}^{\infty} \boldsymbol{E}_{\alpha}\left[\tilde{h}\left(\alpha_{t}\right) ; t \leqq \tau_{n}<+\infty, \sigma_{n}=+\infty\right],
\end{gathered}
$$

and consequently

$$
\lim _{t \rightarrow \infty} \boldsymbol{E}_{\alpha}\left[\tilde{h}\left(\alpha_{t}\right) ; \zeta=+\infty\right]=\sum_{n=1}^{\infty} \boldsymbol{E}_{\alpha}\left[\tilde{h}_{1}\left(\alpha_{\tau_{n}}\right) ; \tau_{n}<+\infty\right] .
$$

Denote the right-hand side of (4.2) by $\tilde{h}_{2}(a)$.

Then

$$
\begin{gathered}
\boldsymbol{E}_{\alpha}\left[\tilde{h}\left(\alpha_{t}\right)\right]=\sum_{k=1}^{|\alpha|} \boldsymbol{E}_{\alpha}\left[h\left(\alpha_{t}\right) ; \zeta_{k}<+\infty, \zeta_{k-1}=+\infty\right] \\
=\sum_{k=1}^{|\alpha|} \boldsymbol{E}_{\alpha}\left[\left.\boldsymbol{E}_{\alpha \xi_{k}}\left[\tilde{h}\left(\alpha_{t-s}\right) ; \zeta=+\infty\right]\right|_{s=\zeta_{k}} ; \zeta_{k}<t\right] \\
+\sum_{k=1}^{|\alpha|} \boldsymbol{E}_{\alpha}\left[\tilde{h}\left(\alpha_{t}\right) ; t \leqq \zeta_{k}<+\infty, \zeta_{k-1}=+\infty\right] .
\end{gathered}
$$

Therefore we get

$$
\lim _{t \rightarrow \infty} \boldsymbol{E}_{\alpha}\left[\tilde{h}\left(\alpha_{t}\right)\right]=\sum_{k=1}^{|\alpha|} \boldsymbol{E}_{\alpha}\left[\tilde{h}_{2}\left(\alpha_{\zeta_{k}}\right) ; \zeta_{k}<+\infty\right] .
$$

Remark. For each $\tilde{h} \in \tilde{\mathscr{H}}$, denote $\Phi \tilde{h}(\alpha)=\lim _{t \rightarrow \infty} \boldsymbol{E}_{\alpha}\left[\tilde{h}\left(\alpha_{t}\right)\right]$. Then $\Phi$ is a map from $\widetilde{\mathscr{H}}$ to $\mathscr{G}$. Furthermore it should be noted that we did not use the condition of Case II in the above proof. Thus Lemma 4.2 holds generally.

Lemma 4.3 For each $g \in \mathscr{G}, \lim _{t \rightarrow \infty} \tilde{\boldsymbol{E}}_{\alpha}\left[g\left(\alpha_{t}\right)\right]$ exists. 
Proof. For $\alpha \in I \backslash \Delta, \quad \tilde{\boldsymbol{E}}_{\alpha}\left[g\left(\alpha_{t}\right) ; \sigma_{1}=+\infty\right]=\boldsymbol{E}_{\alpha}\left[g\left(\alpha_{t}\right) ; \sigma_{1}=+\infty\right]=\boldsymbol{E}_{\alpha}\left[g\left(\alpha_{t}\right)\right]-$ $\boldsymbol{E}_{\alpha}\left[g\left(\alpha_{t}\right) ; \sigma_{1}<+\infty\right]$.

By an argument similar to Lemma 4.2, we have

$$
\lim _{t \rightarrow \infty} \tilde{\boldsymbol{E}}_{\alpha}\left[g\left(\alpha_{t}\right) ; \sigma_{1}=+\infty\right]=g(\alpha)-\boldsymbol{E}_{\alpha}\left[g\left(\alpha_{\sigma_{1}}\right) ; \sigma_{1}<+\infty\right] .
$$

Denote the right-hand side of (4.4) by $g_{1}(\alpha)$. Then

$$
\begin{aligned}
\tilde{\boldsymbol{E}}_{\alpha}\left[g\left(\alpha_{t}\right)\right]=\sum_{n=1}^{\infty} \tilde{\boldsymbol{E}}_{\alpha}\left[g\left(\alpha_{t}\right) ; \tau_{n}<+\infty, \sigma_{n}=+\infty\right](\text { by Lemma 4.1) } \\
=\sum_{n=1}^{\infty} \tilde{\boldsymbol{E}}_{\alpha}\left[\left.\tilde{\boldsymbol{E}}_{\alpha_{\tau_{n}}}\left[g\left(\alpha_{t-s}\right) ; \sigma_{1}=+\infty\right]\right|_{s=\tau_{n}} ; \tau_{n}<t\right]+ \\
\sum_{n=1}^{\infty} \boldsymbol{E}_{\alpha}\left[g\left(\alpha_{t}\right) ; t \leqq \tau_{n}<+\infty, \sigma_{n}=+\infty\right]
\end{aligned}
$$

Thus we have

$$
\lim _{t \rightarrow \infty} \tilde{\boldsymbol{E}}_{\alpha}\left[g\left(\alpha_{t}\right)\right]=\sum_{n=1}^{\infty} \tilde{\boldsymbol{E}}_{\alpha}\left[g_{1}\left(\alpha_{\tau_{n}}\right) ; \tau_{n}<+\infty\right] .
$$

For each $g \in \mathscr{G}$, we denote $\Psi g(\alpha)=\lim _{t \rightarrow \infty} \boldsymbol{E}_{\alpha}\left[g\left(\alpha_{t}\right)\right]$. Then $\Psi$ is a map from $\mathscr{G}$ to $\widetilde{\mathscr{H}}$.

Lemma 4.4 $\Phi$ is a bijection from $\widetilde{\mathscr{H}}$ to $\mathscr{G}$ and $\Phi^{-1}=\Psi$.

Proof. Let $g \in \mathscr{G}$ and $\tilde{h}=\Psi g$. We shall show $\Phi \tilde{h}=g$. Let us calculate $\tilde{h}_{1}$ and $\tilde{h}_{2}$ in the proof of Lemma 4.2. Let $\alpha \in I \backslash \Delta$. By (4.1) and (4.5) we have

$$
\begin{aligned}
\tilde{h}_{1}(\alpha) & =\Psi g(\alpha)-\tilde{\boldsymbol{E}}_{\alpha}\left[\Psi g\left(\alpha_{\sigma_{1}}\right) ; \sigma_{1}<+\infty\right] \\
& =\sum_{n=1}^{\infty}\left(\tilde{\boldsymbol{E}}_{\alpha}\left[g_{1}\left(\alpha_{\tau_{n}}\right) ; \tau_{n}<+\infty\right]-\tilde{\boldsymbol{E}}_{\alpha}\left[\sum_{n=1}^{\infty} \tilde{\boldsymbol{E}}_{\alpha_{\sigma_{1}}}\left[g_{1}\left(\alpha_{\tau_{n}}\right) ; \tau_{n}<+\infty\right] ; \sigma_{1}<+\infty\right]\right. \\
& =\sum_{n=1}^{\infty}\left(\tilde{\boldsymbol{E}}_{\alpha}\left[g_{1}\left(\alpha_{\tau_{n}}\right) ; \tau_{n}<+\infty\right]-\tilde{\boldsymbol{E}}_{\alpha}\left[g_{1}\left(\alpha_{\tau_{n+1}}\right) ; \tau_{n+1}<+\infty\right]\right) \\
& =g_{1}(\alpha)
\end{aligned}
$$

and

$$
\begin{aligned}
\tilde{h}_{2}(\alpha) & =\sum_{n=1}^{\infty} \boldsymbol{E}_{\alpha}\left[\tilde{h}_{1}\left(\alpha_{\tau_{n}}\right) ; \tau_{n}<+\infty\right]=\sum_{n=1}^{\infty} \boldsymbol{E}_{\alpha}\left[g_{1}\left(\alpha_{\tau_{n}}\right) ; \tau_{n}<+\infty\right] \\
& =\sum_{n=1}^{\infty}\left(\boldsymbol{E}_{\alpha}\left[g\left(\alpha_{\tau_{n}}\right) ; \tau_{n}<+\infty\right]-\boldsymbol{E}_{\alpha}\left[\boldsymbol{E}_{\alpha_{\tau_{n}}}\left[g\left(\alpha_{\sigma_{1}}\right) ; \sigma_{1}<+\infty\right] ; \tau_{n}<+\infty\right]\right) \\
& =\sum_{n=1}^{\infty}\left(\boldsymbol{E}_{\alpha}\left[g\left(\alpha_{\tau_{n}}\right) ; \tau_{n}<+\infty\right]-\boldsymbol{E}_{\alpha}\left[g\left(\alpha_{\sigma_{n}}\right) ; \sigma_{n}<+\infty\right]\right) \\
& =\sum_{n=1}^{\infty}\left(\lim _{t \rightarrow \infty} \boldsymbol{E}_{\alpha}\left[g\left(\alpha_{t}\right) ; \sigma_{n}=+\infty\right]-\lim _{t \rightarrow \infty} \boldsymbol{E}_{\alpha}\left[g\left(\alpha_{t}\right) ; \tau_{n}=+\infty\right]\right) \\
& =\lim _{t \rightarrow \infty} \boldsymbol{E}_{\alpha}\left[g\left(\alpha_{t}\right) ; \tau_{n}<+\infty, \quad \text { and } \sigma_{n}=+\infty \text { for some } n \geqq 1\right] \\
& =\lim _{t \rightarrow \infty} \boldsymbol{E}_{\alpha}\left[g\left(\alpha_{t}\right) ; \zeta=+\infty\right] \quad(\text { by Lemma 3.1) } \\
& =g(\alpha)-\boldsymbol{E}_{\alpha}\left[g\left(\alpha_{\zeta}\right) ; \zeta<+\infty\right] .
\end{aligned}
$$


Thus it follows from (4.3) that

$$
\begin{aligned}
\Phi \tilde{h}(\alpha) & =\sum_{k=1}^{|\alpha|} \boldsymbol{E}_{\alpha}\left[\tilde{h}_{2}\left(\alpha_{\zeta_{k}}\right) ; \zeta_{k}<+\infty\right] \\
& =\sum_{k=1}^{|\alpha|} \boldsymbol{E}_{\alpha}\left[g\left(\alpha_{\zeta_{k}}\right) ; \zeta_{k}<+\infty\right]-\sum_{k=1}^{|\alpha|} \boldsymbol{E}_{\alpha}\left[\boldsymbol{E}_{\alpha_{\zeta_{k}}}\left[g\left(\alpha_{\zeta}\right) ; \zeta<+\infty\right] ; \zeta_{k}<+\infty\right] \\
& =\sum_{k=1}^{|\alpha|} \boldsymbol{E}_{\alpha}\left[g\left(\alpha_{\zeta_{k}}\right) ; \zeta_{k}<+\infty\right]-\sum_{k=1}^{|\alpha|} \boldsymbol{E}_{\alpha}\left[g\left(\alpha_{\zeta_{k-1}}\right) ; \zeta_{k-1}<+\infty\right] \\
& =\boldsymbol{E}_{\alpha}\left[g\left(\alpha_{\zeta_{|\alpha|}}\right) ; \zeta_{|\alpha|}<+\infty\right]=g(\alpha) .
\end{aligned}
$$

Conversely, let $\tilde{h} \in \tilde{\mathscr{H}}$ and $g=\Phi \tilde{h}$. For $\alpha \in I \backslash \Delta$,

$$
\begin{aligned}
\lim _{t \rightarrow \infty} & \boldsymbol{E}_{\alpha}\left[g\left(\alpha_{t}\right) ; \sigma_{1}=+\infty\right]=\lim _{t \rightarrow \infty} \boldsymbol{E}_{\alpha}\left[\lim _{s \rightarrow \infty} \boldsymbol{E}_{\alpha_{t}}\left[\tilde{h}\left(\alpha_{s}\right)\right] ; \sigma_{1}=+\infty\right] \\
= & \lim _{t \rightarrow \infty} \lim _{s \rightarrow \infty} \boldsymbol{E}_{\alpha}\left[\boldsymbol{E}_{\alpha_{t}}\left[\tilde{h}\left(\alpha_{s}\right)\right] ; \sigma_{1}>t\right]=\lim _{t \rightarrow \infty} \lim _{s \rightarrow \infty} \boldsymbol{E}_{\alpha}\left[\tilde{h}\left(\alpha_{t+s}\right) ; \sigma_{1}>t\right] \\
= & \lim _{t \rightarrow \infty} \lim _{s \rightarrow \infty} \boldsymbol{E}_{\alpha}\left[\tilde{h}\left(\alpha_{t+s}\right) ; \sigma_{1}=+\infty\right]=\tilde{h}_{1}(\alpha) .
\end{aligned}
$$

Accordingly we have

$$
\begin{aligned}
\Psi g(\alpha)= & \lim _{t \rightarrow \infty} \tilde{\boldsymbol{E}}_{\alpha}\left[g\left(\alpha_{t}\right)\right] \\
= & \lim _{t \rightarrow \infty} \sum_{n=1}^{\infty} \tilde{\boldsymbol{E}}_{\alpha}\left[g\left(\alpha_{t}\right) ; \tau_{n}<+\infty, \sigma_{n}=+\infty\right] \quad \text { (by Lemma 4.1) } \\
= & \lim _{t \rightarrow \infty} \sum_{n=1}^{\infty} \tilde{\boldsymbol{E}}_{\alpha}\left[\tilde{\boldsymbol{E}}_{\alpha_{\tau_{n}}}\left[g\left(\alpha_{t-u} ; \sigma_{1}=+\infty\right]_{u=\tau_{n}} ; \tau_{n}<t\right]\right. \\
& +\lim _{t \rightarrow \infty} \sum_{n=1}^{\infty} \tilde{\boldsymbol{E}}_{\alpha}\left[g\left(\alpha_{t}\right) ; t \leqq \tau_{n}<+\infty, \sigma_{n}=+\infty\right] \\
= & \sum_{n=1}^{\infty} \tilde{\boldsymbol{E}}_{\alpha}\left[\tilde{h}_{1}\left(\alpha_{\tau_{n}}\right) ; \tau_{n}<+\infty\right] \\
= & \sum_{n=1}^{\infty}\left(\tilde{\boldsymbol{E}}_{\alpha}\left[\tilde{h}\left(\alpha_{\tau_{n}}\right) ; \tau_{n}<+\infty\right]-\tilde{\boldsymbol{E}}_{\alpha}\left[\tilde{h}\left(\alpha_{\sigma_{n}}\right) ; \sigma_{n}<+\infty\right]\right) \\
= & \lim _{t \rightarrow \infty} \tilde{\boldsymbol{E}}_{\alpha}\left[\tilde{h}\left(\alpha_{t}\right) ; \tau_{n}<+\infty, \sigma_{n}=+\infty \quad \text { for some } \quad n \geqq 1\right] \\
= & \tilde{h}(\alpha) \quad(\text { by Lemma } 4.1) .
\end{aligned}
$$

Thus we complete the proof of Lemma 4.4.

The following lemma is a slight modification of Lemma 3.1 of Matloff [7]. His original proof can be applied also in this case.

Lemma 4.5 Let $\left\{t_{n}\right\}$ be an increasing sequence tending to $\infty$ and let $\left\{f_{n}\right\}$ be a sequence of functions on $S$ such that

(i) $0 \leqq f_{n} \leqq 1$ for all $n$, and

(ii) $\bar{f}(i)=\lim _{n \rightarrow \infty}\left(P_{t_{n}} f_{n}\right)(i)$ exists for each $i \in S$.

Then $\bar{f}$ is $Q$-harmonic. 
Corollary 4.6 Let $\left\{f_{t}(i)\right\}_{t>0}$ be a family of functions on $S$ such that

(i) $0 \leqq f_{t} \leqq 1$ for each $t>0$, and

(ii) $g(i)=P_{t} f_{t}(i)$ for each $t>0$ and $i \in S$.

Then $g \in \mathscr{H}$.

Lemma 4.7 Let $\tilde{h} \in \tilde{\mathscr{H}}$. For arbitrarily fixed $\alpha \in I$, set $h_{\alpha}(i)=\tilde{h}\left(\alpha+\varepsilon^{i}\right)$. Then $h_{\alpha} \in \mathscr{H}$.

Proof. Noting (2.4), we have

$$
h_{\alpha}(i)=\tilde{h}\left(\alpha+\varepsilon^{i}\right)=\tilde{\boldsymbol{E}}_{\alpha+\varepsilon^{i}}\left[\tilde{h}\left(\alpha_{t}\right)\right]=\sum_{j \in S} P_{t}(i, j) \tilde{\boldsymbol{E}}_{\alpha}\left[\tilde{h}\left(\alpha_{t}+\varepsilon^{j}\right)\right] .
$$

Therefore $h_{\alpha} \in \mathscr{H}$ by Corollary 4.6.

Remark. Lemma 4.7 is interesting in itself, and it is generalized as follows. Let $\left(X_{t}, P_{x}^{1}\right)$ and $\left(Y_{t}, P_{y}^{2}\right)$ be two Markov processes on state spaces $\left(X, \mathscr{F}^{1}\right)$ and $\left(Y, \mathscr{F}^{2}\right)$, which are generated by bounded infinitesimal generators and let $\left(\left(X_{t}, Y_{t}\right)\right.$, $\left.P_{(x, y)}=P_{x} \otimes P_{y}\right)$ be their direct product Markov process. If a bounded measurable function $f(x, y)$ on $\left(X \times Y, \mathscr{F}^{1} \times \mathscr{F}^{2}\right)$ satisfies that $E_{(x, y)}\left[f\left(X_{t}, Y_{t}\right)\right]=f(x, y)$ for every $(x, y) \in X \times Y$ and all $t>0$, then it holds that $E_{x}^{1}\left[f\left(X_{t}, y\right)\right]=f(x, y)$ and $E_{y}^{2}\left[f\left(x, Y_{t}\right)\right]=f(x, y)$ for every $(x, y) \in X \times Y$ and all $t>0$.

Lemma 4.8 Let $f_{i, j}=\tilde{\boldsymbol{P}}_{\varepsilon^{i}+\varepsilon^{j}}\left[\sigma_{1}<+\infty\right]$. Then, we have

$$
\lim _{t \rightarrow \infty} \sum_{j \in S} P_{t}(i, j) f_{j, k}=0 \quad \text { for each } i \text { and } k \text { of } S \text {. }
$$

Proof. For each $\delta>0$, set $f_{i j}^{\delta}=\tilde{\boldsymbol{P}}_{\varepsilon^{i}+\varepsilon^{j}}\left[\alpha_{n \delta} \in \Delta\right.$ for some $\left.n \geqq 1\right]$. Note that $\tilde{\boldsymbol{P}}_{\varepsilon^{i}+\varepsilon^{j}}\left[\sum_{n=1}^{\infty} I_{\Delta}\left(\alpha_{n \delta}\right)<+\infty\right]=1$ by the condition of Case II. Then we have

$$
\begin{aligned}
f_{i, j}^{\delta} & =\sum_{n=1}^{\infty} \sum_{m \in S} \tilde{\boldsymbol{P}}_{\varepsilon^{i}+\varepsilon^{j}}\left[\alpha_{n \delta}=2 \varepsilon^{m}\right]_{2} \tilde{\boldsymbol{P}}_{\varepsilon^{m}}\left[\alpha_{n \delta} \in I \backslash \Delta \text { for all } n \geqq 1\right] \\
& =\sum_{n=1}^{\infty} \sum_{m \in S} P_{n \delta}(i, m) P_{n \delta}(j, m)\left(1-f_{m, m}^{\delta}\right)
\end{aligned}
$$

Let $J$ be any finite subset of $S$, and $N$ be any positive integer. Then,

$$
\begin{aligned}
& \left(f_{i, j}^{\delta}-\sum_{n=1}^{N} \sum_{m \in J} P_{n \delta}(i, m) P_{n \delta}(j, m)\left(1-f_{m, m}^{\delta}\right)\right)^{2} \\
& \quad \leqq\left(\sum_{(n, m): n>N \text { or } m \notin J} P_{n \delta}(i, m)^{2}\left(1-f_{m, m}^{\delta}\right)\right)\left(\sum_{n=1}^{\infty} \sum_{m \in S} P_{n \delta}(j, m)^{2}\left(1-f_{m, m}^{\delta}\right)\right) \\
& \quad=\left(\sum_{(n, m): n>N \text { or } m \xi J} P_{n \delta}(i, m)^{2}\left(1-f_{m, m}^{\delta}\right)\right) f_{j, j}^{\delta} .
\end{aligned}
$$

Here we used the Schwarz's inequality and (4.6).

For any $\varepsilon>0$ there exist $J$ and $N$ such that

$$
\sum_{(n, m): n>N \text { or } m \notin J} P_{n \delta}^{2}(i, m)\left(1-f_{m, m}^{\delta}\right)<\varepsilon^{2} .
$$

Thus, we get 


$$
f_{i, j}^{\delta} \leqq \sum_{n=1}^{N} \sum_{m \in J} P_{n \delta}(i, m) P_{n \delta}(j, m)+\varepsilon \quad \text { for any } j \in S .
$$

So,

$$
\sum_{j \in S} P_{t}(i, j) f_{j, k}^{\delta} \leqq \sum_{n=1}^{N} \sum_{m \in J} P_{n \delta}(k, m) P_{t+n \delta}(i, m)+\varepsilon .
$$

Since the condition of Case II implies that $\left\{P_{t}(i, j)\right\}$ is not positively recurrent, it is well-known that

$$
\lim _{t \rightarrow \infty} P_{t}(i, j)=0 \text { for any } i \text { and } j \in S \text {. }
$$

Hence we obtain $\lim _{t \rightarrow \infty} \sum_{j \in S} P_{t}(i, j) f_{j, k}^{\delta}=0$.

Also for $i \neq j$ and $\delta>0$

$$
\begin{aligned}
f_{i, j} & =\tilde{\boldsymbol{P}}_{\varepsilon^{i}+\varepsilon^{j}}\left[\sigma_{1}<+\infty\right]=\tilde{\boldsymbol{P}}_{\varepsilon^{i}+\varepsilon^{j}}\left[\sigma_{1}<+\infty, \eta_{1}\left(\vartheta_{\sigma_{1}}\right) \leqq \delta\right]+\tilde{\boldsymbol{P}}_{\varepsilon^{i+\varepsilon^{j}}}\left[\sigma_{1}<+\infty, \quad \eta_{1}\left(\vartheta_{\sigma_{1}}\right)>\delta\right] \\
& \leqq \tilde{\boldsymbol{E}}_{\varepsilon^{i}+\varepsilon^{j}}\left[\tilde{\boldsymbol{P}}_{\alpha_{\sigma_{1}}}\left[\eta_{1} \leqq \delta\right] ; \sigma_{1}<+\infty\right]+f_{i, j}^{\delta} \\
& \leqq \sup _{|\alpha|=2} \tilde{\boldsymbol{P}}_{\alpha}\left[\eta_{1} \leqq \delta\right]+f_{i, j}^{\delta}=\sup _{|\alpha| \leqq 2}\left(1-\exp \left(-\left|R_{\alpha, \alpha}\right| \cdot \delta\right)\right)+f_{i, j}^{\delta} .
\end{aligned}
$$

Noting $\sup _{|\alpha|=2}\left|R_{\alpha, \alpha}\right|<+\infty$, we can conclude that

$$
\lim _{t \rightarrow \infty} \sum_{j \in S} P_{t}(i, j) f_{j, k}=0 \text { for any } i \text { and } k \text { of } S \text {. }
$$

Lemma 4.9 For any $\mu \in \mathscr{S}$, set $g(\alpha)=\left\langle\mu, f_{\alpha}\right\rangle$ and $\Psi g(\alpha)=\tilde{h}(\alpha)$. Then for each $\alpha \in I$ and $k \in S$,

$$
\lim _{t \rightarrow \infty} \lim _{s \rightarrow \infty} \tilde{\boldsymbol{E}}_{\alpha}\left[\boldsymbol{E}_{\alpha_{t}}\left[\tilde{h}\left(\alpha_{s}+\varepsilon^{k}\right)\right]\right]=\tilde{h}\left(\alpha+\varepsilon^{k}\right) .
$$

Proof. $1^{\circ}$. Let us consider a Markov chain $\left(\left(\alpha_{t}^{\prime}, \varepsilon_{t}^{\prime}\right), P_{(\alpha, \varepsilon)}^{\prime}\right)$ which satisfies the following conditions.

(i) $\left(\left(\alpha_{t}^{\prime}, \varepsilon_{t}^{\prime}\right), P_{(\alpha, \varepsilon)}^{\prime}\right)$ is a Markov chain on $I^{\prime}=I \times\{\alpha \in I ;|\alpha| \leqq 1\}$,

(ii) $\left(\alpha_{t}^{\prime}, P_{(\alpha, \varepsilon)}^{\prime}\right)$ is identical with $\left(\alpha_{t}, \boldsymbol{P}_{\alpha}\right)$ in the sense of probability law, and

(iii) $\left(\alpha_{t}^{\prime}+\varepsilon_{t}^{\prime}, P_{(\alpha, \varepsilon)}^{\prime}\right)$ is identical with $\left(\alpha_{t}, \boldsymbol{P}_{\alpha+\varepsilon}\right)$ in the sense of probability law.

In fact, $\left(\left(\alpha_{t}^{\prime}, \varepsilon_{t}^{\prime}\right), P_{(\alpha, \varepsilon)}^{\prime}\right)$ can be constructed by the following infinitesimal matrix,

$$
R_{\left(\alpha, \varepsilon^{k}\right), \beta}^{\prime}= \begin{cases}\alpha_{i} q_{i j} & \text { if } \beta=\left(\alpha-\varepsilon^{i}+\varepsilon^{j}, \varepsilon^{k}\right) \quad(i \neq j) \\ q_{k j} & \text { if } \beta=\left(\alpha, \varepsilon^{j}\right) \quad(j \neq k) \\ \frac{1}{4 N} \alpha_{i}\left(\alpha_{i}-1\right) & \text { if } \beta=\left(\alpha-\varepsilon^{i}, \varepsilon^{k}\right) \\ \frac{1}{2 N} \alpha_{k} & \text { if } \beta=(\alpha, 0) \\ \sum_{i \in S} \alpha_{i} q_{i i}+q_{k k}-\frac{1}{4 N} \sum_{i \in S} \alpha_{i}\left(\alpha_{i}-1\right)-\frac{1}{2 N}|\alpha| \text { if } \beta=\left(\alpha, \varepsilon^{k}\right) \\ 0 & \text { otherwise, }\end{cases}
$$


and

$$
R_{(\alpha, 0), \beta}^{\prime}= \begin{cases}\alpha_{i} q_{i j} & \text { if } \beta=\left(\alpha-\varepsilon^{i}+\varepsilon^{j}, 0\right) \quad(i \neq j) \\ \frac{1}{4 N} \alpha_{i}\left(\alpha_{i}-1\right) & \text { if } \beta=\left(\alpha-\varepsilon^{i}, 0\right) \\ \sum_{i \in S} \alpha_{i} q_{i i}-\frac{1}{4 N} \sum_{i \in S} \alpha_{i}\left(\alpha_{i}-1\right) & \text { if } \beta=(\alpha, 0) \\ 0 & \text { otherwise. }\end{cases}
$$

$2^{\circ}$. Next, we claim that

$$
\boldsymbol{P}_{\alpha} \otimes \boldsymbol{P}_{\varepsilon^{k}}[\bar{\tau} \leqq t] \leqq \tilde{\boldsymbol{P}}_{\alpha} \otimes \boldsymbol{P}_{\varepsilon^{k}}[\bar{\tau} \leqq t], \quad \text { where } \quad \bar{\tau}=\inf \left\{t \geqq 0 ; \alpha_{t} \geqq \varepsilon_{t}\right\} .
$$

In order to show (4.9) it is sufficient to construct a Markov chain $\left(\left(\bar{\alpha}_{t}, \bar{\beta}_{t}\right), \bar{P}_{(\alpha, \beta)}\right)$ such that

(i ) $\left(\left(\bar{\alpha}_{t}, \bar{\beta}_{t}\right), \bar{P}_{(\alpha, \beta)}\right)$ is a Markov chain on $\bar{I}=\{(\alpha, \beta) \in I \times I ; \alpha \leqq \beta\}$,

(ii) $\left(\bar{\alpha}_{t}, \bar{P}_{(\alpha, \beta)}\right)$ has the identical probability law with $\left(\alpha_{t}, \boldsymbol{P}_{\alpha}\right)$ for every $\beta \in I$ with $\alpha \leqq \beta$,

and

(iii) $\left(\bar{\beta}_{t}, \bar{P}_{(\alpha, \beta)}\right)$ has the identical probability law with $\left(\alpha_{t}, \tilde{\boldsymbol{P}}_{\beta}\right)$ for every $\alpha \in I$ with $\alpha \leqq \beta$.

The infinitesimal matrix of $\left(\left(\bar{\alpha}_{t}, \bar{\beta}_{t}\right), \bar{P}_{(\alpha, \beta)}\right)$ is given by

$$
\bar{R}_{(\alpha, \beta),\left(\alpha^{\prime}, \beta^{\prime}\right)}=\left\{\begin{array}{lr}
\alpha_{i} q_{i j} & \text { if } \quad\left(\alpha^{\prime}, \beta^{\prime}\right)=\left(\alpha-\varepsilon^{i}+\varepsilon^{j}, \beta-\varepsilon^{i}+\varepsilon^{j}\right) \quad(i \neq j) \\
\left(\beta_{i}-\alpha_{i}\right) q_{i j} & \text { if } \quad\left(\alpha^{\prime}, \beta^{\prime}\right)=\left(\alpha, \beta-\varepsilon^{i}+\varepsilon^{j}\right) \quad(i \neq j) \\
\frac{1}{4 N} \alpha_{i}\left(\alpha_{i}-1\right) & \text { if } \quad\left(\alpha^{\prime}, \beta^{\prime}\right)=\left(\alpha-\varepsilon^{i}, \beta\right) \\
\sum_{i \in S} \beta_{i} q_{i i}-\frac{1}{4 N} \sum_{i \in S} \alpha_{i}\left(\alpha_{i}-1\right) & \text { if } \quad\left(\alpha^{\prime}, \beta^{\prime}\right)=(\alpha, \beta) \\
0 & \text { otherwise. }
\end{array}\right.
$$

$3^{\circ}$. By Lemma 4.7 we have

$$
\tilde{\boldsymbol{E}}_{\alpha}\left[\boldsymbol{E}_{\alpha_{t}}\left[\tilde{h}\left(\alpha_{s}+\varepsilon^{k}\right)\right]\right]=\tilde{\boldsymbol{E}}_{\alpha} \otimes \boldsymbol{E}_{\varepsilon^{k}}\left[\boldsymbol{E}_{\alpha_{t}} \otimes \boldsymbol{E}_{\varepsilon_{t}}\left[\tilde{h}\left(\alpha_{s}+\varepsilon_{s}\right)\right]\right] .
$$

$4^{\circ}$. It follows from Lemma 4.2, Lemma 4.3 and Lemma 4.4 that for $h=\Psi g$

$$
\begin{aligned}
\tilde{h}\left(\alpha+\varepsilon^{k}\right) & =\lim _{t \rightarrow \infty} \tilde{\boldsymbol{E}}_{\alpha+\varepsilon^{k}}\left[\lim _{s \rightarrow \infty} \boldsymbol{E}_{\alpha_{t}}\left[\tilde{h}\left(\alpha_{s}\right)\right]\right] . \\
& =\lim _{t \rightarrow \infty} \lim _{s \rightarrow \infty} \tilde{\boldsymbol{E}}_{\alpha} \otimes \boldsymbol{E}_{\varepsilon^{k}}\left[\boldsymbol{E}_{\alpha_{t}+\varepsilon_{t}}\left[\tilde{h}\left(\alpha_{s}\right)\right]\right. \\
& =\lim _{t \rightarrow \infty} \lim _{s \rightarrow \infty} \tilde{\boldsymbol{E}}_{\alpha} \otimes \boldsymbol{E}_{\varepsilon^{k}}\left[E_{\left(\alpha_{t}, \varepsilon_{t}\right)}^{\prime}\left[\tilde{h}\left(\alpha_{s}^{\prime}+\varepsilon_{s}^{\prime}\right)\right]\right]
\end{aligned}
$$

$5^{\circ}$. Obviously we have 


$$
E_{\left(\alpha, \varepsilon^{k}\right)}^{\prime}\left[\tilde{h}\left(\alpha_{s}^{\prime}+\varepsilon_{s}^{\prime}\right) ; s<\bar{\tau}\right]=\boldsymbol{E}_{\alpha} \otimes \boldsymbol{E}_{\varepsilon^{k}}\left[\tilde{h}\left(\alpha_{s}+\varepsilon_{s}\right) ; s<\bar{\tau}\right]
$$

$6^{\circ}$. Hence it follows that

$$
\begin{aligned}
& \mid \lim _{t \rightarrow \infty} \lim _{s \rightarrow \infty} \tilde{\boldsymbol{E}}_{\alpha}\left[\boldsymbol{E}_{\alpha_{t}}\left[\tilde{h}\left(\alpha_{s}+\varepsilon^{k}\right)\right]-\tilde{h}\left(\alpha+\varepsilon^{k}\right) \mid\right. \\
& =\lim _{t \rightarrow \infty} \lim _{s \rightarrow \infty} \mid \tilde{\boldsymbol{E}}_{\alpha} \otimes \boldsymbol{E}_{\varepsilon^{k}}\left[\boldsymbol{E}_{\alpha_{t}} \otimes \boldsymbol{E}_{\varepsilon_{t}}\left[\tilde{h}\left(\alpha_{s}+\varepsilon\right)\right]\right] \\
& -\tilde{\boldsymbol{E}}_{\alpha} \otimes \boldsymbol{E}_{\varepsilon^{k}}\left[E_{\left(\alpha_{t}, \varepsilon_{t}\right)}^{\prime}\left[h\left(\alpha_{s}^{\prime}+\varepsilon_{s}^{\prime}\right)\right]\right] \mid \quad(\text { by }(4.11) \text { and (4.12)) } \\
& =\lim _{t \rightarrow \infty} \lim _{s \rightarrow \infty} \mid \tilde{\boldsymbol{E}}_{\alpha} \otimes \boldsymbol{E}_{\varepsilon^{k}}\left[\boldsymbol{E}_{\alpha_{t}} \otimes \boldsymbol{E}_{\varepsilon_{t}}\left[\tilde{h}\left(\alpha_{s}+\varepsilon_{s}\right) ; s \geqq \bar{\tau}\right]\right] \\
& -\tilde{\boldsymbol{E}}_{\alpha} \otimes \boldsymbol{E}_{\varepsilon^{k}}\left[E_{\left(\alpha_{t}, \varepsilon_{t}\right)}^{\prime}\left[\tilde{h}\left(\alpha_{s}^{\prime}+\varepsilon_{s}^{\prime}\right) ; s \geqq \bar{\tau}\right]\right] \mid \\
& \leqq \lim _{t \rightarrow \infty} \lim _{s \rightarrow \infty} \tilde{\boldsymbol{E}}_{\alpha} \otimes \boldsymbol{E}_{\varepsilon^{k}}\left[\boldsymbol{P}_{\alpha_{t}} \otimes \boldsymbol{P}_{\varepsilon_{t}}[\bar{\tau} \leqq s]\right] \\
& \leqq \lim _{t \rightarrow \infty} \lim _{s \rightarrow \infty} \tilde{\boldsymbol{E}}_{\alpha} \otimes \boldsymbol{E}_{\varepsilon^{k}}\left[\tilde{\boldsymbol{P}}_{\alpha_{t}} \otimes \boldsymbol{P}_{\varepsilon_{t}}[\bar{\tau} \leqq s]\right] \quad \text { (by (4.9)) } \\
& \leqq \lim _{t \rightarrow \infty} \lim _{s \rightarrow \infty} \sum_{j: \varepsilon^{j} \leqq \alpha} \tilde{\boldsymbol{P}}_{\varepsilon^{j}+\varepsilon^{k}}\left[\alpha_{u} \in \Delta \text { for some } t \leq u<t+s\right] \\
& \leqq \sum_{j: \varepsilon^{j} \leqq \alpha} \boldsymbol{P}_{\varepsilon^{j+\varepsilon^{k}}}\left[\alpha_{t_{n}} \in \Delta \text { for some indreasing sequence }\left\{t_{n}\right\} \text {, tending to } \infty\right] \\
& =0 \text {. }
\end{aligned}
$$

Thus we complete the proof of Lemma 4.9.

Now we can prove Theorem 1.3.

$1^{\circ}$. For each $h \in \mathscr{H}$, we put $\tilde{h}(\alpha)=\prod_{i \in S} h(i)^{\alpha_{i}}$. Then $\tilde{h} \in \mathscr{H}$. By Lemma 4.2, $\lim _{t \rightarrow \infty}\left\langle T_{t}^{*} \delta_{h}, f_{\alpha}\right\rangle=\lim _{t \rightarrow \infty} \boldsymbol{E}_{\alpha}\left[\tilde{h}\left(\alpha_{t}\right)\right]$ exists for each $\alpha \in I$.

Hence there exists a probability measure $v_{h}$ on $X$ such that

$$
\lim _{t \rightarrow \infty} T_{t}^{*} \delta_{h}=v_{h} \text {. }
$$

$2^{\circ} .\left\langle T_{t}^{*} \delta_{h}, f_{\varepsilon^{i}}\right\rangle=\boldsymbol{E}_{\varepsilon^{i}}\left[\tilde{h}\left(\alpha_{t}\right)\right]=P_{t} h(i)=h(i)$.

(ii) follows from this.

$3^{\circ}$. Let $\mu \in \mathscr{S}_{\text {ex }}$. Since $v_{0}=\delta_{0}$ and $v_{1}=\delta_{1}$, we assume $\mu \neq \delta_{0}, \delta_{1}$. Then for some $k \in S, 0<\int \mu(d x) x_{k}<1$ holds. But we can see easily that $0<\int \mu(d x) x_{k}<1$ holds for all $k \in S$.

Let any $k \in S$ be fixed. We define two probability measures $\mu_{1}$ and $\mu_{2}$ by

$$
\left\{\begin{aligned}
\left\langle\mu_{1}, f\right\rangle & =\int \mu(d x) f(x) x_{k} / \int \mu(d x) x_{k} \\
\left\langle\mu_{2}, f\right\rangle & =\int \mu(d x) f(x)\left(1-x_{k}\right) / \int \mu(d x)\left(1-x_{k}\right), \\
& \text { for all } f \in C(X) .
\end{aligned}\right.
$$


Then it holds that $\mu=\lambda \mu_{1}+(1-\lambda) \mu_{2}$ for $\lambda=\int \mu(d x) x_{k}$.

First we claim

$$
\lim _{t \rightarrow \infty} \frac{1}{t} \int_{0}^{t} T_{s}^{*} \mu_{1} d s=\mu .
$$

Suppose that for some increasing sequence $\left\{t_{n}\right\}$ tending to $\infty, \lim _{n \rightarrow \infty} \frac{1}{t_{n}} \int_{0}^{t_{n}} T_{s}^{*} \mu_{1} d s$ converges to $\bar{\mu} \in \mathscr{P}(X)$. Then $\lim _{n \rightarrow \infty} \frac{1}{t_{n}} \int_{0}^{t_{n}} T_{s}^{*} \mu_{2} d s=\bar{\mu}$ exists and ${ }^{n \rightarrow \infty} \mu=\lambda \bar{\mu}+(1-\lambda) \bar{\mu}$ holds. Since $\bar{\mu} \in \mathscr{S}, \vec{\mu} \in \mathscr{S}$, and $\mu \in \mathscr{S}_{\text {ex }}$, we have $\bar{\mu}=\bar{\mu}=\mu$. Hence it follows that $\lim _{t \rightarrow \infty} \int_{0}^{t} T_{s}^{*} \mu_{1} d s=\mu$ because of the compactness of $\mathscr{P}(X)$.

$4^{\circ}$. Let $g(\alpha)=\left\langle\mu, f_{\alpha}\right\rangle(\alpha \in I)$. Then $\mu \in \mathscr{S}$ implies that $g \in \mathscr{G}$. Then we have

$$
\lim _{t \rightarrow \infty} \frac{1}{t} \int_{0}^{t} d s \boldsymbol{E}_{\alpha}\left[g\left(\alpha_{s}+\varepsilon^{k}\right)\right]=g\left(\varepsilon^{k}\right) g(\alpha) .
$$

Indeed, noting that $\left\langle\mu_{1}, f_{\alpha}\right\rangle=g\left(\alpha+\varepsilon^{k}\right) / g\left(\varepsilon^{k}\right)$, we obtain (4.16) by using (4.15) and Lemma 3.1.

$5^{\circ}$. Next, we claim that for $\tilde{h}=\Psi g$

$$
\lim _{u \rightarrow \infty} \lim _{t \rightarrow \infty} \frac{1}{t} \int_{0}^{t} \tilde{\boldsymbol{E}}_{\alpha}\left[\boldsymbol{E}_{\alpha_{u}}\left[g\left(\alpha_{s}+\varepsilon^{k}\right)\right]\right] d s=\tilde{h}\left(\alpha+\varepsilon^{k}\right)
$$

It follows from Lemma 4.4 that

$$
\begin{aligned}
\tilde{\boldsymbol{E}}_{\alpha}\left[\boldsymbol{E}_{\alpha_{u}}\left[g\left(\alpha_{s}+\varepsilon^{k}\right)\right]\right] & =\tilde{\boldsymbol{E}}_{\alpha}\left[\boldsymbol{E}_{\alpha_{u}}\left[\lim _{v \rightarrow \infty} \boldsymbol{E}_{\alpha_{s}+\varepsilon^{k}}\left[\tilde{h}\left(\alpha_{v}\right)\right]\right]\right] \\
& =\lim _{v \rightarrow \infty} \tilde{\boldsymbol{E}}_{\alpha}\left[\boldsymbol{E}_{\alpha_{u}}\left[E_{\left(\alpha_{s}, \varepsilon^{k}\right)}^{\prime}\left[\tilde{h}\left(\alpha_{v}^{\prime}+\varepsilon_{v}^{\prime}\right)\right]\right]\right]
\end{aligned}
$$

Noting that $E_{\left(\alpha, \varepsilon^{k}\right)}^{\prime}\left[\tilde{h}\left(\alpha_{v}^{\prime}+\varepsilon_{v}^{\prime}\right) ; v<\bar{\tau}\right]=\boldsymbol{E}_{\alpha} \otimes \boldsymbol{E}_{\varepsilon^{k}}\left[\tilde{h}\left(\alpha_{v}+\varepsilon_{v}\right) ; v<\bar{\tau}\right]$ we see

$$
\begin{aligned}
& \varlimsup_{v \rightarrow \infty} \mid \tilde{\boldsymbol{E}}_{\alpha}\left[\boldsymbol{E}_{\alpha_{u}}\left[E_{\left(\alpha_{s}, \varepsilon^{k}\right)}^{\prime}\left[\tilde{h}\left(\alpha_{v}^{\prime}+\varepsilon_{v}^{\prime}\right)\right]-\boldsymbol{E}_{\alpha_{s}} \otimes \boldsymbol{E}_{\varepsilon^{k}}\left[\tilde{h}\left(\alpha_{v}+\varepsilon_{v}\right)\right]\right] \mid\right. \\
& \quad \leqq \varlimsup_{v \rightarrow \infty} \tilde{\boldsymbol{E}}_{\alpha}\left[\boldsymbol { E } _ { \alpha _ { u } } \left[\boldsymbol{P}_{\alpha_{s}} \otimes \boldsymbol{P}_{\varepsilon^{k}}[\bar{\tau} \leqq v]\right.\right. \\
& \quad=\tilde{\boldsymbol{E}}_{\alpha}\left[\boldsymbol{P}_{\alpha_{u}} \otimes \boldsymbol{P}_{\varepsilon^{k}}\left[\alpha_{t+s} \geqq \varepsilon_{t} \text { for some } t \geqq 0\right]\right. \\
& \leqq \tilde{\boldsymbol{E}}_{\alpha}\left[\tilde{\boldsymbol{P}}_{\alpha_{u}} \otimes \boldsymbol{P}_{\varepsilon^{k}}\left[\alpha_{t+s} \geqq \varepsilon_{t} \text { for some } t \geqq 0\right]\right. \\
& \leqq \tilde{\boldsymbol{E}}_{\alpha}\left[\sum_{j: \varepsilon^{j} \leqq \alpha_{u+s}} f_{j . k}\right]
\end{aligned}
$$

Here we notice that these inequalities are justified rigorously by making use of the process $\left(\left(\bar{\alpha}_{t}, \bar{\beta}_{t}\right), P_{(\alpha, \beta)}\right)$.

On the other hand it follows from Lemma 4.7 that

$$
\begin{aligned}
\tilde{\boldsymbol{E}}_{\alpha} & {\left[\boldsymbol{E}_{\alpha_{u}}\left[\boldsymbol{E}_{\alpha_{s}} \otimes \boldsymbol{E}_{\varepsilon^{k}}\left[\tilde{h}\left(\alpha_{v}+\varepsilon_{v}\right)\right]\right]\right]=\tilde{\boldsymbol{E}}_{\alpha}\left[\boldsymbol{E}_{\alpha_{u}}\left[\boldsymbol{E}_{\alpha_{s}}\left[\tilde{h}\left(\alpha_{v}+\varepsilon^{k}\right)\right]\right]\right] } \\
& =\tilde{\boldsymbol{E}}_{\alpha}\left[\boldsymbol{E}_{\alpha_{u}}\left[\tilde{h}\left(\alpha_{s+v}+\varepsilon^{k}\right)\right]\right] .
\end{aligned}
$$

Thus by (4.18), (4.19) and (4.20), we have 


$$
\begin{aligned}
& \left.\mid \tilde{\boldsymbol{E}}_{\alpha}\left[\boldsymbol{E}_{\alpha_{u}}\left[g\left(\alpha_{s}+\varepsilon^{k}\right)\right]\right]-\lim _{v \rightarrow \infty} \tilde{\boldsymbol{E}}_{\alpha_{u}}\left[\tilde{h}\left(\alpha_{v}+\varepsilon^{k}\right)\right]\right] \mid \\
& \quad \leqq \tilde{\boldsymbol{E}}_{\alpha}\left[\sum_{j: \varepsilon^{j} \leqq \alpha_{u+s}} f_{j, k}\right] .
\end{aligned}
$$

Hence by using Lemma 4.8, Lemma 4.9 and (4.21), we obtain

$$
\begin{aligned}
& \left|\lim _{u \rightarrow \infty} \lim _{t \rightarrow \infty} \frac{1}{t} \int_{0}^{t} \tilde{\boldsymbol{E}}_{\alpha}\left[\boldsymbol{E}_{\alpha_{u}}\left[g\left(\alpha_{s}+\varepsilon^{k}\right)\right]\right] d s-\tilde{h}\left(\alpha+\varepsilon^{k}\right)\right| \\
& \quad=\left|\lim _{u \rightarrow \infty} \lim _{t \rightarrow \infty} \frac{1}{t} \int_{0}^{t} \tilde{\boldsymbol{E}}_{\alpha}\left[\boldsymbol{E}_{\alpha_{u}}\left[g\left(\alpha_{s}+\varepsilon^{k}\right)\right]\right] d s-\lim _{u \rightarrow \infty} \lim _{v \rightarrow \infty} \tilde{\boldsymbol{E}}_{\alpha}\left[\boldsymbol{E}_{\alpha_{u}}\left[\tilde{h}\left(\alpha_{v}+\varepsilon^{k}\right)\right]\right]\right| \\
& \quad \leqq \varlimsup_{u \rightarrow \infty} \varlimsup_{t \rightarrow \infty} \frac{1}{t} \int_{0}^{t}\left|\tilde{\boldsymbol{E}}_{\alpha}\left[\boldsymbol{E}_{\alpha_{u}}\left[g\left(\alpha_{s}+\varepsilon^{k}\right)\right]\right]-\lim _{v \rightarrow \infty} \tilde{\boldsymbol{E}}_{\alpha}\left[\boldsymbol{E}_{\alpha_{u}}\left[\tilde{h}\left(\alpha_{v}+\varepsilon^{k}\right)\right]\right]\right| d s \\
& \quad \leqq \varlimsup_{u \rightarrow \infty} \varlimsup_{t \rightarrow \infty} \frac{1}{t} \int_{0}^{t} \tilde{\boldsymbol{E}}_{\alpha}\left[\sum_{j: \varepsilon^{j} \leqq \alpha_{u+s}} f_{j . k}\right] d s=0
\end{aligned}
$$

$6^{\circ}$. By (4.16) and (4.17), we have

$$
\tilde{h}\left(\alpha+\varepsilon^{k}\right)=\Psi g(\alpha) g\left(\varepsilon^{k}\right)=\tilde{h}(\alpha) g\left(\varepsilon^{k}\right) .
$$

Setting $g\left(\varepsilon^{k}\right)=h(k)$, we see easily that

$$
h \in \mathscr{H} \text { and } \tilde{h}(\alpha)=\prod_{i \in S} h(i)^{\alpha_{i}} .
$$

Therefore we can conclude that $\mu=v_{h}$. Thus we see.

$$
\mathscr{S}_{\text {ex }} \subset\left\{v_{h} ; h \in \mathscr{H}\right\} .
$$

Conversely let any $h_{0} \in \mathscr{H}$ be fixed. Then by the Choquet's representation theorem and (4.24), there exists a probability measure $m(d h)$ on $\mathscr{H}$ such that

$$
v_{h_{0}}=\int_{\mathscr{X}} v_{h} m(d h)
$$

Let $g^{h}(\alpha)=\left\langle v_{h}, f_{\alpha}\right\rangle$. Then $g^{h} \in \mathscr{G}$ and $\Psi g^{h}(\alpha)=\prod_{i \in S} h(i)^{\alpha_{i}}$ by Lemma 4.4. Accordingly by $(4.25)$ we have

$$
\prod_{i \in S} h_{0}(i)^{\alpha_{i}}=\int_{\mathscr{C}} \prod_{i \in S} h(i)^{\alpha_{i}} m(d h) \quad \text { for every } \alpha \in I .
$$

Now, let us introduce the following topology on $\mathscr{H}$. Let $\left\{h_{n}\right\}$ be a sequence of $\mathscr{H} . \quad\left\{h_{n}\right\}$ converges to a $h \in \mathscr{H}$ if and only $\lim _{n \rightarrow \infty} h_{n}(i)=h(i)$ for all $i \in S$. Then $\mathscr{H}$ is compact metrizable. Let us denote by $C(\mathscr{H})$ the set of all continuous functions on $\mathscr{H}$. For each $\alpha \in I$, set $F_{\alpha}(h)=\prod_{i \in S} h(i)^{\alpha_{i}}$. Then $F_{\alpha} \in C(\mathscr{H})$ and the linear hull of $\left\{F_{\alpha} ; \alpha \in I\right\}$ is dense in $C(\mathscr{H})$ with the uniform norm by the Stone-Weierstrass's theorem. Hence by (4.26) we have

$$
F\left(h_{0}\right)=\int_{\mathscr{C}} F(h) m(\mathrm{~d} h) \text { for all } F \in C(\mathscr{H}) .
$$


(4.27) implies $m=\delta_{\left\{h_{0}\right\}}$, that is, the point mass at $h_{0}$. Hence $v_{h_{0}} \in \mathscr{S}_{\text {ex }}$. Thus we complete the proof of Theorem 1.3.

\section{Ergodic theorems for Case II}

This section is devoted to the proof of Theorem 1.4.

$1^{\circ}$. Suppose that for $\mu \in \mathscr{P}(X), T_{t}^{*} \mu$ converges to $v_{h}$ as $t \rightarrow \infty$ for some $h \in \mathscr{H}$. Set $g(\alpha)=\left\langle\mu, f_{\alpha}\right\rangle$ and $g^{h}(\alpha)=\left\langle v_{h}, f_{\alpha}\right\rangle$. Then by Lemma 3.1 we have

$$
\lim _{t \rightarrow \infty} \tilde{\boldsymbol{E}}_{\alpha}\left[g\left(\alpha_{t}\right)\right]=g^{h}(\alpha) \quad \text { for every } \quad \alpha \in I .
$$

For $\alpha \in I \backslash \Delta$, it follows from (5.1) that

$$
\begin{aligned}
& \lim _{t \rightarrow \infty} \tilde{\boldsymbol{E}}_{\alpha}\left[g\left(\alpha_{t}\right) ; \sigma_{1}=+\infty\right]=\lim _{t \rightarrow \infty} \boldsymbol{E}_{\alpha}\left[g\left(\alpha_{t}\right) ; \sigma_{1}=+\infty\right] \\
& \quad=\lim _{t \rightarrow \infty} \boldsymbol{E}_{\alpha}\left[g\left(\alpha_{t}\right)\right]-\lim _{t \rightarrow \infty} \boldsymbol{E}_{\alpha}\left[g\left(\alpha_{t}\right) ; \sigma_{1}<+\infty\right] \\
& \quad=g^{h}(\alpha)-\boldsymbol{E}_{\alpha}\left[g^{h}\left(\alpha_{\sigma_{1}}\right) ; \sigma_{1}<+\infty\right] .
\end{aligned}
$$

We have

$$
\begin{aligned}
\tilde{\boldsymbol{E}}_{\alpha}\left[g\left(\alpha_{t}\right)\right]= & \sum_{n=1}^{\infty} \tilde{\boldsymbol{E}}_{\alpha}\left[g\left(\alpha_{t}\right) ; \tau_{n}<+\infty, \sigma_{n}=+\infty\right] \quad \text { (by Lemma 4.1) } \\
= & \sum_{n=1}^{\infty} \tilde{\boldsymbol{E}}_{\alpha}\left[\left.\tilde{\boldsymbol{E}}_{\alpha_{\tau_{n}}}\left[g\left(\alpha_{t-u}\right) ; \sigma_{1}=+\infty\right]\right|_{u=\tau_{n}} ; \tau_{n}<t\right] \\
& +\sum_{n=1}^{\infty} \tilde{\boldsymbol{E}}_{\alpha}\left[g\left(\alpha_{t}\right) ; t \leqq \tau_{n}<+\infty, \sigma_{n}=+\infty\right],
\end{aligned}
$$

Accordingly we get by (5.2)

$$
\lim _{t \rightarrow \infty} \tilde{\boldsymbol{E}}_{\alpha}\left[g\left(\alpha_{t}\right)\right]=\sum_{n=1}^{\infty} \tilde{\boldsymbol{E}}_{\alpha}\left[g^{h}\left(\alpha_{\tau_{n}}\right)-\boldsymbol{E}_{\alpha_{\tau_{n}}}\left[g^{h}\left(\alpha_{\sigma_{1}}\right) ; \sigma_{1}<+\infty\right] ; \tau_{n}<+\infty\right]
$$

In particular (5.3) is valid for $g=g^{h}$. Thus we have

$$
\begin{aligned}
\lim _{t \rightarrow \infty} \tilde{\boldsymbol{E}}_{\alpha}\left[g\left(\alpha_{t}\right)\right] & =\lim _{t \rightarrow \infty} \boldsymbol{E}_{\alpha}\left[g^{h}\left(\alpha_{t}\right)\right] \\
& =\prod_{i S \epsilon} h(i)^{\alpha_{i}} \quad(\text { by Lemma } 4.4) .
\end{aligned}
$$

Furthermore we can see easily that (5.4) is equivalent to

(5.5) $\sum_{j \in S} P_{t}(i, j) x_{j}$ converges to $h(i)$ as $t \rightarrow \infty$, in probability with respect to $\mu$.

Conversely let us assume (5.5). Then (5.4) holds. We obtain easily that

$$
\lim _{t \rightarrow \infty} \boldsymbol{E}_{\alpha}\left[g\left(\alpha_{t}\right) ; \sigma_{1}=+\infty\right]=\tilde{h}(\alpha)-\tilde{\boldsymbol{E}}_{\alpha}\left[\tilde{h}\left(\alpha_{\sigma_{1}}\right) ; \sigma_{1}<+\infty\right]
$$

for $\alpha \in I \backslash \Delta$, where $\tilde{h}(\alpha)=\prod_{i \in S} h(i)^{\alpha_{i}}$. 
Thus we have

$$
\lim _{t \rightarrow \infty} \boldsymbol{E}_{\alpha}\left[g\left(\alpha_{t}\right) ; \sigma_{1}=+\infty\right]=\lim _{t \rightarrow \infty} \boldsymbol{E}_{\alpha}\left[\tilde{h}\left(\alpha_{t}\right) ; \sigma_{1}=+\infty\right],
$$

and

$$
\lim _{t \rightarrow \infty} \boldsymbol{E}_{\alpha}\left[g\left(\alpha_{t}\right)\right]=\lim _{t \rightarrow \infty} \boldsymbol{E}_{\alpha}\left[\tilde{h}\left(\alpha_{t}\right)\right]
$$

This implies $\lim _{t \rightarrow \infty} T_{t}^{*} \mu=v_{h}$.

Corollary 5.1 Suppose that $\mu \in \mathscr{P}(X)$ satisfies the following condition,

$$
<\mu, f_{\alpha}>=\prod_{i \in S} \int \mu(d x) x_{i}^{\alpha_{i}} \text { for each } \alpha \in I .
$$

Then $T_{t}^{*} \mu$ converges to $v_{h}$ as $t \rightarrow \infty$ for some $h \in \mathscr{H}$ if and only if

(5.10) $\sum_{j \in S} P_{t}(i, j) m_{j}$ converges to $h(i)$ as $t \rightarrow \infty$ for all $i \in S$, where $m_{j}=\int \mu(d x) x_{j}$.

Proof. Assume (5.10). Then

$$
\begin{aligned}
& \int \mu(d x)\left(\sum_{j \in S} P_{t}(i, j) x_{j}-h(i)\right)^{2}=\int \mu(d x) \sum_{j \neq k} \sum_{t} P_{t}(i, j) P_{t}(i, k) x_{j} x_{k} \\
& \quad+\int \mu(\mathrm{d} x) \sum_{j \in S} P_{t}(i, j)^{2} x_{j}^{2}-2 h(i) \sum_{j \in S} P_{t}(i, j) m_{j}+h(i)^{2} \\
& =\left(\sum_{j \in S} P_{t}(i, j) m_{j}-h(i)\right)^{2}+\sum_{j \in S} P_{t}(i, j)^{2}\left(\int \mu(\mathrm{d} x) x_{j}^{2}-m_{j}^{2}\right)
\end{aligned}
$$

Note that the condition of Case II implies that $\lim _{t \rightarrow \infty} \sum_{j \in S} P_{t}(i, j)^{2}=0$. Hence we have

$$
\lim _{t \rightarrow \infty} \int \mu(\mathrm{d} x)\left(\sum_{j \in S} P_{t}(i, j) x_{j}-h(i)\right)^{2}=0 .
$$

Therefore by Theorem 1.4 we have $\lim _{t \rightarrow \infty} T_{t}^{*} \mu=v_{h}$.

Corollary 5.2 Let $x \in X$. Suppose that $\lim _{t \rightarrow \infty} T_{t}^{*} \delta_{x}$ exists. Then

$$
\lim _{t \rightarrow \infty} T_{t}^{*} \delta_{x}=v_{h} \text { for some } h \in \mathscr{H} .
$$

Proof. It follows immediately that $\lim _{t \rightarrow \infty} \sum_{j \in S} P_{t}(i, j) x_{j}$ exists for all $i \in S$ and (5.5) is fulfilled for $h(i)=\lim _{t \rightarrow \infty} \sum_{j \in S} P_{t}(i, j) x_{j}$.

Corollary 5.3 Let $\mu \in \mathscr{P}(X)$ and let $U$ be an open subset of $\mathscr{P}(X)$ containing $\mathscr{S}$. Then there exists a positive number $t_{0}$ such that $T_{t}^{*} \mu \in U$ for all $t>t_{0}$.

Proof. It suffices to show that if $\left\{T_{t_{n}}^{*} \mu\right\}$ converges to $\bar{\mu} \in \mathscr{P}(X)$, for an increasing sequence $\left\{t_{n}\right\}$ tending to $\infty$, then $\bar{\mu} \in \mathscr{S}$. Set $\left\langle\mu, f_{\alpha}\right\rangle=g(\alpha)$ and $\left\langle\bar{\mu}, f_{\alpha}\right\rangle=$ $\bar{g}(\alpha)$. Then, $\lim _{n \rightarrow \infty} \boldsymbol{E}_{\alpha}\left[g\left(\alpha_{t_{n}}\right)\right]=\bar{g}(\alpha)$. Since we can apply Lemma 4.5 for $\left(\alpha_{t}, \boldsymbol{P}_{\alpha}\right)$, we obtain that $\bar{g} \in \mathscr{G}$. Therefore $\bar{\mu} \in \mathscr{S}$ holds. 


\section{Case III}

In this section we shall investigate an example of Case III, which illustrates an different phenomenon from Case I amd Case II.

Let $S=\{s=(i, n) ; i=1,2, \ldots, k n=1,2, \ldots\} \cup\{0\}(2 \leqq k \leqq+\infty)$, and $Q=\left\{q_{s, s^{\prime}}\right\}$ be defined as follows:

$$
q_{s . s^{\prime}}= \begin{cases}a_{i}>0 & \text { if } \quad s=0 \text { and } s^{\prime}=(i, 1) \\ -\sum_{i=1}^{k} a_{i} & \text { if } \quad s=s^{\prime}=0 \\ \lambda_{n}>0 & \text { if } s=(i, n) \text { and } s^{\prime}=(i, n+1) \\ \mu_{n}>0 & \text { if } \quad s=(i, n) \text { and } s^{\prime}=(i, n-1) \\ -\left(\lambda_{n}+\mu_{n}\right) & \text { if } s=s^{\prime}=(i, n) \\ 0 & \text { otherwise. }\end{cases}
$$

Let us assume

$$
\sup _{n}\left(\lambda_{n}+\mu_{n}\right)<+\infty \text { and } \sum_{i=1}^{\infty} a_{i}<+\infty \text { if } k=\infty .
$$

Then $Q=\left\{q_{s, s^{\prime}}\right\}$ generates a unique continuous time Markov chain $\left(X_{t}, P_{s}\right)_{s \in S}$ satisfying

$$
P_{s}\left[X_{t}=s^{\prime}\right]=P_{t}\left(s, s^{\prime}\right) \quad \text { where } \quad P_{t}=e^{t Q} .
$$

For $s \in S$ let us define $\varphi(s)$ and $|s|$ by $s=(\varphi(s),|s|)$, where if $s=0, \varphi(s)=0$ and $|s|=0$. Then we can see that $\left(\left|X_{t}\right|, P_{s}\right)$ is a birth and death process with a reflecting barrier at the origin associated with $\left\{\lambda_{n}, \mu_{n}\right\}$, where $\lambda_{0}=\sum_{i=1}^{k} a_{i}$.

If the $\left\{\lambda_{n}, \mu_{n}\right\}$-birth and death process is recurrent, $Q$ satisfies the condition of Case $I$ or Case II. So we shall assume that the $\left\{\lambda_{n}, \mu_{n}\right\}$-birth and death process is transient. Then it follows obviously that

$$
P_{s}\left[\lim _{t \rightarrow \infty}\left|X_{t}\right|=+\infty\right]=1 \quad \text { for all } s \in S .
$$

Now, let us consider a compactified space $\bar{S}$ of $S$ by adding $k$ number of infinity points $\left\{1^{\infty}, 2^{\infty}, \ldots, k^{\infty}\right\}$ in such sense that a sequence of $S \supset\left\{s_{n}\right\}$ converges to $i^{\infty}$ if and only if $\lim _{n \rightarrow \infty}\left|s_{n}\right|=+\infty$ and $\lim _{n \rightarrow \infty} \varphi\left(s_{n}\right)=i . \quad(1 \leqq i \leqq k)$

We denote by $\mathscr{H}_{\text {ex }}$ the set of all extremal elements of $\mathscr{H}$. Then we have

Lemma 6.1 Let $h_{i}(s)=P_{s}\left[\lim _{t \rightarrow \infty} X_{t}=i^{\infty}\right]$. Then,

(i) $h_{i} \in \mathscr{H}$ and satisfies that $h_{i}(s)>0$ for all $s \in S$, and $\sum_{n=1}^{k} h_{i}=1$,

(ii) $\lim _{s \rightarrow j^{\infty}} h_{i}(s)=0$ if $j \neq i$, and $\lim _{s \rightarrow i^{\infty}} h_{i}(s)=1$, 
and

(iii) $\mathscr{H}_{e x}=\left\{h_{\beta} \mid \beta \subset\{1,2, \ldots, k\}\right\}$, where $h_{\beta}(s)=\sum_{i \in \beta} h_{i}(s)$ and $h_{\emptyset}=0$.

Proof. Since (i) and (ii) are easy, we will show only (iii). Let $h \in \mathscr{H}$. We can see easily that $h\left(j^{\infty}\right)=\lim _{s \rightarrow j^{\infty}} h(s)$ exists and $h(s)=\sum_{j=1}^{k} h\left(j^{\infty}\right) h_{j}(s)$ for each $s \in S$.

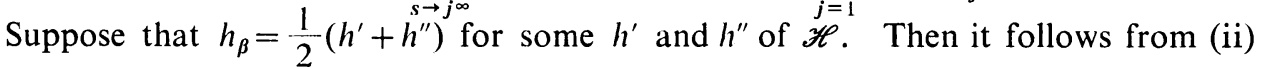
that

$$
h^{\prime}\left(j^{\infty}\right)= \begin{cases}1 & \text { if } j \in \beta \\ 0 & \text { otherwise. Hence } h^{\prime}=h^{\prime \prime}=h_{\beta} . \quad \text { Thus we have } h_{\beta} \in \mathscr{H}_{\text {ex }} .\end{cases}
$$

Next, we shall show the converse. Let $h \in \mathscr{H}_{\text {ex }}$.

Suppose that $0<h\left(i^{\infty}\right)<1$ for some $i$. Then $h(s)$ is represented as follows.

$$
h(s)=h\left(i^{\infty}\right) h_{i}(s)-\left(1-h\left(i^{\infty}\right)\right) h^{\prime}(s) \text { with } h^{\prime}(s)=\frac{h(s)-h\left(i^{\infty}\right) h_{i}(s)}{1-h\left(i^{\infty}\right)} .
$$

Since $h^{\prime} \in \mathscr{H}$ and $h \in \mathscr{H}_{\text {ex }}$, we have $h(s)=h_{i}(s)$ and $h\left(i^{\infty}\right)=1$. So, that is a contradiction. Thus we see that $h\left(j^{\infty}\right)=0$ or 1 for any $j$. Hence $h=h_{\beta}$ for $\beta=\left\{i ; h\left(i^{\infty}\right)=1\right\}$.

Let $\left(\left(X_{t}^{1}, X_{t}^{2}\right), P_{\left(s_{1}, s_{2}\right)}\right)$ be a continuous time Markov chain on $S \times S$ with $P_{t} \otimes P_{t}$ as its transition probability. The following lemma implies that $Q=\left\{q_{s, s^{\prime}}\right\}$ of (6.1) satisfies the condition of Case III.

\section{Lemma 6.2}

$$
0<P_{\left(s s_{2}\right)},\left[\int_{0}^{\infty} I_{\Delta_{2}}\left(X_{t}^{1}, X_{t}^{2}\right) d t=+\infty\right]=\sum_{i=1}^{k} h_{i}\left(s_{1}\right) h_{i}\left(s_{2}\right)<1 \text { for all }\left(s_{1}, s_{2}\right) .
$$

Proof. We refer to Karlin-McGregor's result [4] that $\left\{\lambda_{n}, \mu_{n}\right\}$-birth and death process has the coincidence property almost surely under our assumption. Accordingly we have

$$
P_{\left(s_{1}, s_{2}\right)}\left[\int_{0}^{\infty} I_{0}\left(\left|X_{t}^{1}\right|,\left|X_{t}^{2}\right|\right) d t=+\infty\right]=1 \quad \text { for all }\left(s_{1}, s_{2}\right),
$$

where $I_{0}(n, m)=0$ if $n \neq m$, and $I_{0}(n, n)=1$.

Hence it follows that

$$
\begin{aligned}
& P_{\left(s_{1}, s_{2}\right)}\left[\int_{0}^{\infty} I_{\Delta_{2}}\left(X_{t}^{1}, X_{t}^{2}\right) d t=+\infty\right] \\
& \quad=P_{\left(s_{1}, s_{2}\right)}\left[\int_{0}^{\infty} I_{\Delta_{2}}\left(X_{t}^{1}, X_{t}^{2}\right) d t=+\infty, \lim _{t \rightarrow \infty} X_{t}^{1}=\lim _{t \rightarrow \infty} X_{t}^{2}\right] \\
& \quad=\sum_{i=1}^{k} P_{\left(s_{1}, s_{2}\right)}\left[\int_{0}^{\infty} I_{0}\left(\left|X_{t}^{1}\right|,\left|X_{t}^{2}\right|\right) d t=+\infty, \lim _{t \rightarrow \infty} X_{t}^{1}=\lim _{t \rightarrow \infty} X_{t}^{2}=i^{\infty}\right]
\end{aligned}
$$




$$
\begin{aligned}
& =\sum_{i=1}^{k} P_{\left(s_{1}, s_{2}\right)}\left[\lim _{t \rightarrow \infty} X_{t}^{1}=\lim _{t \rightarrow \infty} X_{t}^{2}=i^{\infty}\right] \\
& =\sum_{i=1}^{k} h_{i}\left(s_{1}\right) h_{i}\left(s_{2}\right) .
\end{aligned}
$$

\section{Lemma 6.3}

(i) $0<\tilde{\boldsymbol{P}}_{\alpha}\left[\int_{0}^{\infty} I_{\Delta}\left(\alpha_{t}\right) d t=+\infty\right]<1$ if $|\alpha| \leqq k$.

(ii) $\tilde{\boldsymbol{P}}_{\alpha}\left[\int_{0}^{\infty} I_{\Delta}\left(\alpha_{t}\right) \mathrm{d} t=+\infty\right]=1$ if $|\alpha| \geqq k+1$.

Proof. Let $\left(\left(X_{t}^{1}, \ldots, X_{t}^{m}\right), P_{\left(s_{1}, \ldots, s_{m}\right)}\right)$ be the direct product Markov process of $m$ number of $P_{t}$-Markov chains on $S$.

For $m \leqq k$ and $\alpha=\varepsilon^{s_{1}}+\cdots+\varepsilon^{s_{m}}$,

$$
\begin{aligned}
& \tilde{\boldsymbol{P}}_{\alpha}\left[\int_{0}^{\infty} I_{\Delta}\left(\alpha_{t}\right) d t<+\infty\right]=P_{\left(s_{1}, \ldots, s_{m}\right)}\left[\int_{0}^{\infty} I_{\Delta}\left(X_{t}^{1}, \ldots, X_{t}^{m}\right) d t<+\infty\right] \\
& \quad \geqq P_{\left(s_{1}, \ldots, s_{m}\right)}\left[\lim _{t \rightarrow \infty} X_{t}^{1}=1^{\infty}, \ldots, \lim _{t \rightarrow \infty} X_{t}^{m}=m^{\infty}\right]=h_{1}\left(s_{1}\right) \cdots h_{m}\left(s_{m}\right)>0 .
\end{aligned}
$$

Hence we have

$$
\tilde{\boldsymbol{P}}_{\alpha}\left[\int_{0}^{\infty} I_{\Delta}\left(\alpha_{t}\right) d t=+\infty\right]<1 \quad \text { for } \quad|\alpha| \leqq k .
$$

For $m \geqq k+1$ and $\alpha=\varepsilon^{s_{1}} \cdots+\varepsilon^{s_{m}}$, it follows from (6.4) that

$$
\begin{aligned}
& \tilde{\boldsymbol{P}}_{\alpha}\left[\int_{0}^{\infty} I_{\Delta}\left(\alpha_{t}\right) d t=+\infty\right]=P_{\left(s_{1}, \ldots, s_{m}\right)}\left[\int_{0}^{\infty} I_{\Delta_{2}}\left(X_{t}^{p}, X_{t}^{q}\right) d t=+\infty,\right. \\
& \text { for some } p \neq q \in\{1, \ldots, m\}] \\
& \geqq P_{\left(s_{1}, \ldots, s_{m}\right)}\left[\int_{0}^{\infty} I_{\Delta_{2}}\left(X_{t}^{p}, X_{t}^{q}\right) d t=+\infty, \lim _{t \rightarrow \infty} X_{t}^{p}=\lim _{t \rightarrow \infty} X_{t}^{q},\right. \\
& \quad \text { for some } p \neq q \in\{1, \ldots, m\}] \\
& =P_{\left(s_{1}, \ldots, s_{m}\right)}\left[\int_{0}^{\infty} I_{0}\left(\left|X_{t}^{p}\right|,\left|X_{t}^{q}\right|\right) d t=+\infty, \lim _{t \rightarrow \infty} X_{t}^{p}=\lim _{t \rightarrow \infty} X_{t}^{q},\right. \\
& \quad \text { for some } p \neq q \in\{1, \ldots, m\}] \\
& =P_{\left(s_{1}, \ldots, s_{m}\right)}\left[\lim _{t \rightarrow \infty} X_{t}^{1}=\lim _{t \rightarrow \infty} X_{t}^{2}, \text { for some } p \neq q \in\{1, \ldots, m\}\right]=1 .
\end{aligned}
$$

We regard an element of $I$ as a bounded counting measure (i.e. integer-valued measure) on $S$. Let us denote by $\bar{I}$ the set of all bounded counting measures on $\bar{S}$ and introduce on $\bar{I}$ the weak topology namely, $\alpha_{n} \rightarrow \alpha$ as $n \rightarrow \infty$ means that $\left\langle\alpha_{n}, f\right\rangle$ $\rightarrow\langle\alpha, f\rangle$ as $n \rightarrow \infty$ for all continuous function $f$ on $\bar{S}$. Let us define $J=\{\alpha \in \bar{I} \mid$ $\alpha(S)=0\}$ and $J_{0}=\left\{\alpha \in J \mid \alpha\left(\left\{i^{\infty}\right\}\right) \leqq 1\right.$ for each $\left.i \in\{1, \ldots, k\}\right\}$. An element $\alpha$ of $J_{0}$ is denoted by $\beta^{\infty}=\left\{i_{1}^{\infty}, \ldots, i_{m}^{\infty}\right\}$ if the support of $\alpha$ is $\left\{i_{1}^{\infty}, \ldots, i_{m}^{\infty}\right\}$ and $\beta=\left\{i_{1}, \ldots, i_{m}\right\}$ $\subset\{1, \ldots, k\}$. 


\section{Lemma 6.4}

(i) $\boldsymbol{P}_{\alpha}\left[\zeta_{k}<+\infty\right]=1$ if $k<+\infty$.

(ii) $\boldsymbol{P}_{\alpha}\left[\lim _{t \rightarrow \infty} \alpha_{t}\right.$ exists in $\left.J_{0}\right]=1$ for every $\alpha \in I$ with $\alpha \neq 0$.

(iii) $\boldsymbol{P}_{\alpha}\left[\lim _{t \rightarrow \infty} \alpha_{t}=\beta^{\infty}\right]>0$ if $\beta^{\infty} \in J_{0}, \alpha \in I$, and $|\beta| \leqq|\alpha| \wedge k$.

Proof. (i): Let $|\alpha| \geqq k+1$. Then it follows from Lemma 6.3 that

$$
\boldsymbol{P}_{\alpha}\left[\sigma_{1}<+\infty\right]=1 \quad \text { if } \quad \alpha \in I \backslash \Delta .
$$

But by Lemma 3.1

$\boldsymbol{P}_{\alpha}[\zeta=+\infty]=\sum_{n=1}^{\infty} \boldsymbol{P}_{\alpha}\left[\tau_{n}<+\infty, \sigma_{n}=+\infty\right]=\sum_{n=1}^{\infty} \boldsymbol{E}_{\alpha}\left[\boldsymbol{P}_{\alpha_{\tau_{n}}}\left[\sigma_{1}=+\infty\right] ; \tau_{n}<+\infty\right]$

Thus we have $\boldsymbol{P}_{\alpha}[\zeta<+\infty]=1$ if $|\alpha| \geqq k+1$, and (i) follows from this.

(ii): First we note that $\tilde{\boldsymbol{P}}_{\alpha}\left[\lim _{t \rightarrow \infty} \alpha_{t}\right.$ exists in $\left.J\right]=1$ by Lemma 6.1 .

For $\alpha \in I \backslash \Delta$ and $|\alpha| \leqq k$, we have

$$
\begin{aligned}
\boldsymbol{P}_{\alpha} & {\left[\sigma_{1}=+\infty\right]=\tilde{\boldsymbol{P}}_{\alpha}\left[\sigma_{1}=+\infty\right]=\tilde{\boldsymbol{P}}_{\alpha}\left[\sigma_{1}=+\infty, \lim _{t \rightarrow \infty} \alpha_{t} \text { exists in } J_{0}\right] } \\
& =\boldsymbol{P}_{\alpha}\left[\sigma_{1}=+\infty, \lim _{t \rightarrow \infty} \alpha_{t} \text { exists in } J_{0}\right],
\end{aligned}
$$

and

$$
\begin{aligned}
\boldsymbol{P}_{\alpha} & {[\zeta=+\infty]=\sum_{n=1}^{\infty} \boldsymbol{P}_{\alpha}\left[\tau_{n}<+\infty, \sigma_{n}=+\infty\right]=\sum_{n=1}^{\infty} \boldsymbol{E}_{\alpha}\left[\boldsymbol{P}_{\alpha_{\tau_{n}}}\left[\sigma_{1}=+\infty\right] ; \tau_{n}<+\infty\right] } \\
& =\sum_{n=1}^{\infty} \boldsymbol{E}_{\alpha}\left[\boldsymbol{P}_{\alpha_{\tau_{n}}}\left[\sigma_{1}=+\infty, \lim _{t \rightarrow \infty} \alpha_{t} \text { exists in } J_{0}\right] ; \tau_{n}<+\infty\right] \\
& =\boldsymbol{P}_{\alpha}\left[\zeta=+\infty, \lim _{t \rightarrow \infty} \alpha_{t} \text { exists in } J_{0}\right] .
\end{aligned}
$$

Accordingly we see

$$
\begin{aligned}
\boldsymbol{P}_{\alpha} & {\left[\lim _{t \rightarrow \infty} \alpha_{t} \text { exists in } J_{0}\right]=\sum_{m=1}^{|\alpha| \wedge k} \boldsymbol{P}_{\alpha}\left[\lim _{t \rightarrow \infty} \alpha_{t} \text { exists in } J_{0}, \zeta_{m}<+\infty, \zeta_{m-1}=+\infty\right] } \\
= & \sum_{m=1}^{|\alpha| \wedge k} \boldsymbol{E}_{\alpha}\left[\boldsymbol{P}_{\alpha_{\zeta_{m}}}\left[\lim _{t \rightarrow \infty} \alpha_{t} \text { exists in } J_{0}, \zeta=+\infty\right] ; \zeta_{m}<+\infty\right] \\
= & \sum_{m=1}^{|\alpha| \wedge k} \boldsymbol{E}_{\alpha}\left[\boldsymbol{P}_{\alpha_{\zeta_{m}}}[\zeta=+\infty] ; \zeta_{m}<+\infty\right]=\sum_{m=1}^{|\alpha| \wedge k} \boldsymbol{P}_{\alpha}\left[\zeta_{m}<+\infty, \zeta_{m-1}=+\infty\right]=1 .
\end{aligned}
$$

(iii): First we note that if $m \leqq k$ and $s_{1}=\left(1, n_{1}\right), \ldots, s_{m}=\left(m, n_{m}\right)$,

$$
\begin{aligned}
& P_{\left(s_{1}, \ldots, s_{m}\right)}\left[\lim _{t \rightarrow \infty} X_{t}^{1}=1^{\infty}, \cdots, \lim _{t \rightarrow \infty} X_{t}^{m}=m^{\infty}, \sigma_{1}=+\infty\right] \\
& \geqq P_{s_{1}}\left[X_{t}^{1} \neq 0 \text { for all } t \geqq 0\right] \cdots P_{s_{m}}\left[X_{t}^{m} \neq 0 \text { for all } t \geqq 0\right]>0 .
\end{aligned}
$$

This implies that for each $\beta^{\infty} \in J_{0}$ and $\alpha \in I$ with $|\alpha|=|\beta|$,

$$
\tilde{\boldsymbol{P}}_{\alpha}\left[\lim _{t \rightarrow \infty} \alpha_{t}=\beta^{\infty}, \sigma_{1}=+\infty\right]>0 .
$$


Let $\alpha \in I$ and $\left|\beta^{\infty}\right|=m \leqq|\alpha| \wedge k$. Then,

$$
\begin{aligned}
& \boldsymbol{P}_{\alpha} {\left[\lim _{t \rightarrow \infty} \alpha_{t}=\beta^{\infty}\right]=\boldsymbol{P}_{\alpha}\left[\zeta_{m}<+\infty, \zeta_{m-1}=+\infty, \lim _{t \rightarrow \infty} \alpha_{t}=\beta^{\infty}\right] } \\
&=\boldsymbol{E}_{\alpha}\left[\boldsymbol{P}_{\alpha_{\zeta_{m}}}\left[\zeta=+\infty, \lim _{t \rightarrow \infty} \alpha_{t}=\beta^{\infty}\right] ; \zeta_{m}<+\infty\right] \\
& \geqq \boldsymbol{E}_{\alpha}\left[\boldsymbol{P}_{\alpha_{\zeta_{m}}}\left[\tau_{1}<+\infty, \sigma_{1}=+\infty, \lim _{t \rightarrow \infty} \alpha_{t}=\beta^{\infty}\right] ; \zeta_{m}<+\infty\right] \\
& \quad=\boldsymbol{E}_{\alpha}\left[\boldsymbol{E}_{\alpha_{\zeta_{m}}}\left[\boldsymbol{P}_{\alpha_{\tau_{1}}}\left[\sigma_{1}=+\infty, \lim _{t \rightarrow \infty} \alpha_{t}=\beta^{\infty}\right] ; \tau_{1}<+\infty\right] ; \zeta_{m}<+\infty\right]
\end{aligned}
$$

It is obvious that

$$
\begin{aligned}
& \boldsymbol{P}_{\alpha_{\tau_{1}}}\left[\sigma_{1}=+\infty, \lim _{t \rightarrow \infty} \alpha_{t}=\beta^{\infty}\right]=\tilde{\boldsymbol{P}}_{\alpha_{\tau_{1}}}\left[\sigma_{1}=+\infty, \lim _{t \rightarrow \infty} \alpha_{t}=\beta^{\infty}\right]>0, \\
& \boldsymbol{P}_{\alpha_{\zeta_{m}}}\left[\tau_{1}<+\infty\right]>0 \text { and } \boldsymbol{P}_{\alpha}\left[\zeta_{m}<+\infty\right]>0
\end{aligned}
$$

Consequently we have $\boldsymbol{P}_{\alpha}\left[\lim _{t \rightarrow \infty} \alpha_{t}=\beta^{\infty}\right]>0$.

Lemma 6.5 Let $g \in \mathscr{G}$. Then for each $\beta^{\infty} \in J_{0}, g\left(\beta^{\infty}\right)=\lim _{\alpha \rightarrow \beta^{\infty}} g(\alpha)$ exsits and

$$
g(\alpha)=\sum_{\beta^{\infty} \in J_{0}} g\left(\beta^{\infty}\right) \boldsymbol{P}_{\alpha}\left[\lim _{t \rightarrow \infty} \alpha_{t}=\beta^{\infty}\right] .
$$

Proof. Let $\beta^{\infty} \in J_{0}$. For every $\alpha \in I,|\alpha| \geqq\left|\beta^{\infty}\right|, \boldsymbol{P}_{\alpha}\left[\lim _{t \rightarrow \infty} \alpha_{t}=\beta^{\infty}\right]>0$ holds by Lemma 6.4. Here we note that $\left\{g\left(\alpha_{t}\right), \boldsymbol{P}_{\alpha}\right\}$ is a bounded martingale and $\boldsymbol{P}_{\alpha}$. $\left[\lim _{t \rightarrow \infty} g\left(\alpha_{t}\right)\right.$ exists $]=1$. This implies that $\lim _{\alpha \rightarrow \beta^{\infty}} g(\alpha)$ exists for each $\beta^{\infty} \in J_{0}$. Also (6.6) is evident.

Now, we can prove the following.

Theorem 6.6 Assume that $k<+\infty$. Then we have

(i) $\mathscr{S}_{\boldsymbol{e x}}=\left\{v_{h} ; h \in \mathscr{H}_{\text {ex }}\right\}$,

and

(ii) Let $\mu \in \mathscr{P}(X)$ and set $f(\alpha)=\left\langle\mu, f_{\alpha}\right\rangle$ for each $\alpha \in I$. Then $\lim _{t \rightarrow \infty} T_{t}^{*} \mu$ converges as $t \rightarrow+\infty$ if and only if

(6.7) $\lim _{\alpha \rightarrow \beta_{\infty}^{\infty}} f(\alpha)$ exists for each $\beta^{\infty} \in J_{0}$.

Proof. Let $h \in \mathscr{H}_{\text {ex }}$. Then $h=h_{\beta}$ for some $\beta \subset\{1, \ldots, k\}$ by Lemma 6.1. Denoting $\tilde{h}_{\beta}(\alpha)=\prod_{i \in S} h_{\beta}(i)^{\alpha_{i}}$, we have

$$
\left\langle v_{h_{\beta}}, f_{\alpha}\right\rangle=\lim _{t \rightarrow \infty} \boldsymbol{E}_{\alpha}\left[\tilde{h}_{\beta}\left(\alpha_{t}\right)\right]=\sum_{\gamma \infty \in J_{0}}\left(\lim _{\alpha \rightarrow \gamma^{\infty}} \tilde{h}_{\beta}(\alpha)\right) \boldsymbol{P}_{\alpha}\left[\lim _{t \rightarrow \infty} \alpha_{t}=\gamma^{\infty}\right] .
$$

Since $\lim _{\alpha \rightarrow \gamma^{\infty}} \tilde{h}_{\beta}(\alpha)=\left\{\begin{array}{ll}1 & \text { if } \gamma \subset \beta \\ 0 & \text { otherwise }\end{array}\right.$, we obtain

$$
\left\langle v_{h_{\beta}}, f_{\alpha}\right\rangle=\sum_{\substack{\gamma \infty \in J_{0} \\ \gamma \in \beta}} \boldsymbol{P}_{\alpha}\left[\lim _{t \rightarrow \infty} \alpha_{t}=\gamma^{\infty}\right]
$$


Suppose that $v_{h_{\beta}}=\frac{1}{2}\left(v^{\prime}+v^{\prime \prime}\right)$ for some $v^{\prime}$ and $v^{\prime \prime} \in S$. Denote $f^{\beta}(\alpha)=\left\langle v_{h_{\beta}}, f_{\alpha}\right\rangle$, $f^{\prime}(\alpha)=\left\langle v^{\prime}, f_{\alpha}\right\rangle$ and $f^{\prime \prime}(\alpha)=\left\langle v^{\prime \prime}, f_{\alpha}\right\rangle$. Then $f^{\beta}(\alpha)=\frac{1}{2}\left(f^{\prime}(\alpha)+f^{\prime \prime}(\alpha)\right)$. Since $f^{\prime}$ and $f^{\prime \prime} \in \mathscr{G}, \lim _{\alpha \rightarrow \gamma^{\infty}} f^{\prime}(\alpha)=f^{\prime}\left(\gamma^{\infty}\right)$ and $\lim _{\alpha \rightarrow \gamma^{\infty}} f^{\prime \prime}(\alpha)=f^{\prime \prime}\left(\gamma^{\infty}\right)$ exist for every $\gamma^{\infty} \in J_{0}$. Moreover by (6.8)

$$
f^{\beta}\left(\gamma^{\infty}\right)=\left\{\begin{array}{ll}
1 & \text { if } \quad \gamma \subset \beta \\
0 & \text { otherwise. }
\end{array} \text { Hence } f^{\prime}\left(\gamma^{\infty}\right)=f^{\prime \prime}\left(\gamma^{\infty}\right)=f^{\beta}\left(\gamma^{\infty}\right)\right. \text { hold }
$$

Thus we have $f^{\prime}=f^{\prime \prime}=f$. This means $v_{h_{\beta}} \in \mathscr{S}_{e x}$.

Next we shall show the converse.

Let $v \in \mathscr{S}$ and set $f(\alpha)=\left\langle v, f_{\alpha}\right\rangle$. Then it follows from Lemma 6.5 that

$$
f(\alpha)=\sum_{\beta \subset\{1, \ldots, k\}} f\left(\beta^{\infty}\right) \boldsymbol{P}_{\alpha}\left[\lim _{t \rightarrow \infty} \alpha_{t}=\beta^{\infty}\right]
$$

By (6.8) and the Möbius inversion formula, we get

$$
\boldsymbol{P}_{\alpha}\left[\lim _{t \rightarrow \infty} \alpha_{t}=\beta^{\infty}\right]=\sum_{\gamma \in \beta}(-1)^{|\beta| \gamma \mid} f^{\gamma}(\alpha)
$$

Then it follows from (6.9) and (6.10) that

$$
f(\alpha)=\sum_{\beta \subset\{1, \ldots, k\}}\left(\sum_{\gamma \subset \beta \subset\{1, \ldots, k\}}(-1)^{|\beta| \gamma \mid} f\left(\beta^{\infty}\right)\right) f^{\gamma}(\alpha) .
$$

Set $\lambda_{\gamma}=\sum_{\gamma \subset \beta \subset\{1, \ldots, k\}}(-1)^{|\beta \backslash \gamma|} f\left(\beta^{\infty}\right)$. Then we have

$$
\lambda_{\gamma} \geqq 0 \quad \text { for each } \quad \gamma \subset\{1 \ldots, k\} \text { and } \sum_{\gamma \subset\{1, \ldots, k\}} \lambda_{\gamma}=1 .
$$

For

$$
\begin{aligned}
\lambda_{\gamma} & =\sum_{\gamma \subset \beta \subset\{1, \ldots, k\}}(-1)^{|\beta| \gamma \mid} \lim _{n \rightarrow \infty} \int v(d x) \prod_{i \in \beta} x_{(i, n)} \\
& =\lim _{n \rightarrow \infty} \int v(d x) \sum_{\gamma \subset \beta \subset\{1, \ldots, k\}}(-1)^{|\beta| \gamma \mid} \prod_{i \in \beta} x_{(i, n)} \\
& =\lim _{n \rightarrow \infty} \int v(d x) \prod_{i \in \gamma} x_{(i, n)} \prod_{j \in\{1, \ldots, k\} \backslash \gamma}\left(1-x_{(j, n)}\right) \geqq 0,
\end{aligned}
$$

and

$$
\sum_{\gamma\{\subset 1, \ldots, k\}} \lambda_{\gamma}=\lim _{n \rightarrow \infty} \int v(d x) \sum_{\gamma \subset\{1, \ldots, k\}} \prod_{i \in \gamma} x_{(i, n)} \prod_{j \in\{1, \ldots, k\} \backslash \gamma}\left(1-x_{(j, n)}\right)=1 .
$$

Accordingly we have $v=\sum_{\gamma \subset\{1, \ldots, k\}} \lambda_{\gamma} v_{h \gamma}$. Thus we obtain (i). Next, we shall show the latter half. Suppose that $\lim _{t \rightarrow \infty} T_{t}^{*} \mu$ exists. Then we can easily see that (6.7) is valid. Conversely we assume $\stackrel{t \rightarrow \infty}{(6.7)}$. Then

$$
\begin{aligned}
\lim _{t \rightarrow \infty}\left\langle T_{t}^{*} \mu, f_{\alpha}\right\rangle & =\lim _{t \rightarrow \infty} \boldsymbol{E}_{\alpha}\left[\left\langle\mu, f_{\alpha_{t}}\right\rangle\right]=\lim _{t \rightarrow \infty} \sum_{\beta^{\infty} \in J_{0}} \boldsymbol{E}_{\alpha}\left[f\left(\alpha_{t}\right) ; \lim _{u \rightarrow \infty} \alpha_{u}=\beta^{\infty}\right] \\
& =\sum_{\beta^{\infty} \in J_{0}} f\left(\beta^{\infty}\right) \boldsymbol{P}_{\alpha}\left[\lim _{t \rightarrow \infty} \alpha_{t}=\beta^{\infty}\right] .
\end{aligned}
$$


Thus $T_{t}^{*} \mu$ converges as $t \rightarrow \infty$.

Remark. It should be noted that $\left\{v_{h} ; h \in \mathscr{H}_{\text {ex }}\right\} \subset \mathscr{S}_{\text {ex }}$ holds for $k=+\infty$.

\section{Examples and discussions}

Let us consider only the case without mutation.

$1^{\circ}$. Let $S$ be a finite set and let us assume that $\left\{q_{i j}\right\}$ is irreducible. Then obviously $\left\{q_{i j}\right\}$ satisfies the condition of Case I. Hence we have $\mathscr{S}_{e x}=\left\{\delta_{\mathbf{0}}, \delta_{1}\right\}$.

$2^{\circ}$. Let $S=\{1,2,3\}$. Assume that $q_{13}=q_{23}=m, q_{11}=q_{22}=-m$ and $q_{12}=q_{31}$ $=q_{32}=q_{33}=0 . \quad(m>0)$. Then the evolution of gene frequencies is given by

$$
\left\{\begin{array}{l}
d x_{1}(t)=a\left(x_{1}(t)\right) d B_{1}(t)+m\left(x_{3}(t)-x_{1}(t)\right) d t \\
d x_{2}(t)=a\left(x_{2}(t)\right) d B_{2}(t)+m\left(x_{3}(t)-x_{2}(t)\right) d t \\
d x_{3}(t)=a\left(x_{3}(t)\right) d B_{3}(t)
\end{array}\right.
$$

where $a(y)=\left\{\begin{array}{ll}\sqrt{y(1-y)} & \text { if } 0 \leqq y \leqq 1 \\ 0 & \text { otherwise }\end{array},\left\{B_{i}(t)\right\}_{i=1,2,3}\right.$ are an independent system of one-dimensional Brownian motions on $(\Omega, \mathscr{F}, P)$. Let $\xi_{3}=\inf \left\{t \geqq 0 ; x_{3}(t)=0\right.$ or 1\}. Then we can see easily that $\xi_{3}<+\infty P$-a.s. and

$$
\left\{\begin{array}{l}
P\left[\lim _{t \rightarrow \infty} x_{1}(t)=\lim _{t \rightarrow \infty} x_{2}(t)=0 \mid x_{3}\left(\xi_{3}\right)=0\right]=1, \quad \text { and } \\
P\left[\lim _{t \rightarrow \infty} x_{1}(t)=\lim _{t \rightarrow \infty} x_{2}(t)=1 \mid x_{3}\left(\xi_{3}\right)=1\right]=1 .
\end{array}\right.
$$

Hence $\mathscr{S}_{\boldsymbol{e x}}=\left\{\delta_{\mathbf{0}}, \delta_{1}\right\}$ and $\lim _{t \rightarrow \infty} T_{t}^{*} \mu=\lambda \delta_{1}+(1-\lambda) \delta_{\mathbf{0}}$, where $\lambda=\int \mu(d \boldsymbol{x}) P\left[x_{3}\left(\xi_{3}\right)\right.$ $=1 \mid \boldsymbol{x}(0)=\boldsymbol{x}]$.

$3^{\circ}$. Let $S=\{1,2,3\}$. Assume that $q_{31}=q_{32}=m, q_{33}=-2 m$, and $q_{i j}=0$ for all other $(i, j)$. Then the evolution of gene frequencies is given by

$$
\left\{\begin{array}{l}
d x_{1}(t)=a\left(x_{1}(t)\right) d B_{1}(t) \\
d x_{2}(t)=a\left(x_{2}(t)\right) d B_{2}(t) \\
d x_{3}(t)=a\left(x_{3}(t)\right) d B_{3}(t)+m\left(x_{1}(t)+x_{2}(t)-2 x_{3}(t)\right) d t
\end{array}\right.
$$

Let $\xi_{i}=\inf \left\{t \geqq 0 ; x_{i}(t)=0\right.$ or 1$\}(i=1,2)$. Then $\xi_{1}<+\infty, \xi_{2}<+\infty P$-a.e. and

$$
\begin{aligned}
& P\left[\lim _{t \rightarrow \infty} x_{3}(t)=0 \mid x_{1}\left(\xi_{1}\right)=x_{2}\left(\xi_{2}\right)=0\right]=1, \\
& P\left[\lim _{t \rightarrow \infty} x_{3}(t)=1 \mid x_{1}\left(\xi_{1}\right)=x_{2}\left(\xi_{2}\right)=1\right]=1,
\end{aligned}
$$

and the limiting distribution of $\left\{x_{3}(t)\right\}$ under $P\left[\cdot \mid x_{1}\left(\xi_{1}\right) \neq x_{2}\left(\xi_{2}\right)\right]$ is given by the following probability density function,

$$
p_{m}(y)= \begin{cases}C_{m}(x(1-y))^{2 m-1} & \text { if } 0 \leqq y \leqq 1 \\ 0 & \text { otherwise }\end{cases}
$$


where $C_{m}$ is the normalizing constant.

Hence $\mathscr{S}_{e x}=\left\{\delta_{0}, \delta_{1}, v_{1}, v_{2}\right\}$, where $v_{1}$ and $v_{2}$ are defined by

$$
\begin{aligned}
& \left\langle v_{1}, f\right\rangle=\int_{0}^{1} f(1,0, y) p_{m}(y) d y \\
& \left\langle v_{2}, f\right\rangle=\int_{0}^{1} f(0,1, y) p_{m}(y) d y, \text { for all } f \in C\left(\left[\begin{array}{ll}
0 & 1
\end{array}\right]^{3}\right) .
\end{aligned}
$$

Thus $\mathscr{S}_{\text {ex }}=\left\{v_{h} ; h \in \mathscr{H}_{\text {ex }}\right\}$ holds.

$4^{\circ}$. Generally if $Q=\left\{q_{i j}\right\}$ is irreducible and if every $Q$-harmonic function is constant, $Q$ satisfies the condition of Case $I$ or Case $I I$. Let $S=Z^{d}$ (d-dimensional integer lattice space) and let

$$
q_{i j}= \begin{cases}m & \text { if } \quad|i-j|=1 \\ -2 d m & \text { if } i=j \\ 0 & \text { otherwise. }\end{cases}
$$

Then the corresponding $P_{t}=e^{t Q}$ is the $d$-dimensional simple random walks. If $d=1$ or 2 , then Case $I$ holds and $\mathscr{S}_{e x}=\left\{\delta_{0}, \delta_{1}\right\}$, and if $d \geqq 3$, then Case II holds and $\mathscr{S}_{\text {ex }}=\left\{v_{c} ; 0 \leqq c \leqq 1\right\}$.

$5^{\circ}$. Let us consider the case that $\left\{q_{i j}\right\}$ is symmetric. Then we can see easily that if the corresponding $P_{t}$-Markov chain is transient, then Case II holds, and if it is positively recurrent, then Case $I$ holds. However even if it is null recurrent there are examples of Case II. (cf. Liggett [6])

$6^{\circ}$. It is possible to construct a strongly continuous Markov semi-group $\left\{T_{t}\right\}$ on $C(X)$ associated with (1.1) without using stochastic differential equations. We shall show an outline of such a construction. Let $L_{0}$ be the set of all functions satisfying that

(i) $f=\sum_{\alpha \in I} c_{\alpha} f_{\alpha}$ where $c_{\alpha} \in R^{1}$ and $\sum_{\alpha \in I}\left|c_{\alpha}\right|<+\infty$,

and

(ii) there exists a constant $M>0$ such that $c_{\alpha}=0$ if $|\alpha|>M$.

Then we can see that every $f \in L_{0}$ is twice continuously differentiable on $X$ and $A f$ of (1.5) is well-defined. Also $\left(A, D(A)=L_{0}\right)$ is dissipative as an operator on $C(X)$. Hence it is closable. We denote by $(\bar{A}, D(\bar{A}))$ the closure of $(A, D(A))$.

Next, let us consider the following equation,

$$
(\lambda-A) u=f \text { where } \lambda>0 \text { and } f \in L_{0} .
$$

For $f=\sum_{\alpha \in I} c_{\alpha} f_{\alpha}$, set $u(x)=\sum_{\alpha \in I} c_{\alpha} \int_{0}^{\infty} E_{\alpha}\left[f_{\alpha_{t}}(x) \exp \left(-\lambda t-u \int_{0}^{t}\left|\alpha_{s}\right| d s\right)\right] d t$. Then it is easy to see that $u \in L_{0}$ and $u$ satisfies the equation (7.7) by the Feymnan-Kac's theorem. Thus we see that the range of $\lambda-A$ is dense in $C(X)$. Hence by virtue of the Hille-Yosida's semi-group theory, there exists a unique strongly continuous semi-group $\left\{T_{t}\right\}$ such that the infinitesimal generator of $\left\{T_{t}\right\}$ coincides with $(\bar{A}, D(\bar{A}))$. 
Department OF MATHEMATICS

NARA WOMEN's UNIVERSITY

\section{References}

[1] W. H. Fleming \& C. Su, Some one dimensional migration models in population genetics theory. Theor. Popul. Biol. 5, 431-449, (1974).

[2] S. Itazu, Ergodic properties of the equilibrium measure of the stepping stone model in population genetics, (to appear).

[3] K. Ito, Stochastic processes, Tata lecture notes, Bombay, (1962).

[4] S. Karlin \& J. McGregor, Coincidence properties of birth and death processes, Pacific Jour. Math. 9, 1109-1140, (1959).

[5] M. Kimura \& G. H. Weiss, The stepping stone model of population structure and decrease of genetical correlation with distance, Genetics, 49, 561-576, (1964).

[6] T. Liggett, A characterization of the invariant measures for an infinite particle system with interactions, I, II, Trans. Amer. Math. Soc. 179, 433-453, (1973), 198. 201-213, (1974).

[7] N. Matloff, Ergodicity conditions for dissonant voting model, Ann. Prob. 5, 371-386, (1977).

[8] T. Maruyama, Stochastic problems in population genetics, Lecture Notes in Biomathematics, 17, Springer-Verlag, (1977).

[9] T. Nagylaki, Random genetic drift in a cline, Proc. Nat. Acad. Sci. USA, Vol. 75, No. 1, 423-426, (1978).

[10] T. Shiga \& A. Shimizu, Infinite dimensional stochastic differential equations and their applications, (to appear).

[11] F. Spitzer, Recurrent random walk of an infinite particle system, Trans. Amer. Math. Soc. 198, 191-199, (1974). 